OECDpublishing

\title{
ANALYSE DE L'IMPACT
}

DES POLITIQUES FISCALES

ET DE PROTECTION SOCIALE

SUR LES INÉGALITÉS

ET LA PAUVRETÉ AU TOGO

\section{DOCUMENTS D'O
DE L'OCDE SUR \\ LE DÉVELOPPEMENT}

Septembre $2018 \mathbf{n}^{\circ} \mathbf{1 2}$ 
Documents d'orientation de l'OCDE sur le développement

Septembre $2018 \mathrm{n}^{\circ} 12$

\section{ANALYSE DE L'IMPACT DES POLITIQUES FISCALES ET DE PROTECTION SOCIALE SUR LES INÉGALITÉS ET LA PAUVRETÉ AU TOGO}

Par Jon Jellema et Caroline Tassot 
Ce document est publié sous la responsabilité du Secrétaire général de l'OCDE. Les opinions et les interprétations qui y sont exprimées et les arguments qui y sont employés ne reflètent pas nécessairement les vues officielles des gouvernements des pays membres de l'OCDE ou de son Centre de développement.

Ce document, ainsi que les données et cartes qu'il peut comprendre, sont sans préjudice du statut de tout territoire, de la souveraineté s'exerçant sur ce dernier, du tracé des frontières et limites internationales, et du nom de tout territoire, ville ou région.

Ce document a été autorisé par Mario Pezzini, Directeur du Centre de développement et Conseiller spécial auprès du Secrétaire général de l'OCDE sur le développement.

Mots clés : Politique fiscale, Dépenses publiques, Taxation

Codes JEL : E62, H50, H20

Crédit photo : Couverture réalisée par le Centre de développement de l’OCDE

(C) OCDE 2018 


\section{Remerciements}

L'analyse de l'impact des politiques fiscales et de protection sociale sur les inégalités et la pauvreté au Togo a été préparée par l'Unité Cohésion Sociale du Centre de développement de l'OCDE, dans le cadre du programme de l'Union européenne sur les Systèmes de Protection Sociale (EUSPS) en partenariat avec le Commitment to Equity (CEQ) Institute.

L'équipe était dirigée par Alexandre Kolev, Chef de l'Unité Cohésion Sociale, et Ji-Yeun Rim, coordinatrice du programme EUSPS, sous la direction de Mario Pezzini, Directeur du Centre de développement de l'OCDE et Conseiller spécial du Secrétaire général de l'OCDE sur le développement.

L'analyse de l'impact des politiques fiscales et de protection sociale sur les inégalités et la pauvreté au Togo a été coordonnée et élaborée par Caroline Tassot et Jon Jellema. Le rapport a été examiné par Alexandre Kolev, Ji-Yeun Rim, Naoko Ueda (Centre de développement de l'OCDE) et Maya Goldman (CEQ). Le rapport a également bénéficié de commentaires précieux de la part de Marta Palmarola Adrados (Délégation de l'Union européenne au Togo), James Browne et Herwig Immervoll (Direction de l'Emploi, du travail et des affaires sociales). 


\section{Résumé}

Le gouvernement togolais a entrepris des réformes importantes après la crise des années 90 dans le but d'améliorer les conditions de vie de la population grâce à des politiques publiques adaptées, notamment à travers la Stratégie de croissance accélérée et de promotion de l'emploi (SCAPE) 2013-17. Malgré des améliorations du niveau d'éducation et une augmentation de l'espérance de vie, les défis en termes de pauvreté et d'inégalités restent très importants. Cette étude estime l'impact redistributif des revenus fiscaux (les impôts) et des dépenses fiscales à travers les transferts monétaires, non monétaires, et les subventions, sur les revenus des ménages et les inégalités. Deux conclusions ressortent de cette analyse : la politique fiscale réduit les inégalités au Togo, mais appauvrit les ménages les plus pauvres. 


\section{Préface}

Le gouvernement togolais a entrepris des réformes importantes après la crise des années 90 dans le but d'améliorer les conditions de vie de la population grâce à des politiques publiques adaptées, notamment à travers la Stratégie de croissance accélérée et de promotion de l'emploi (SCAPE) 2013-17. Malgré des améliorations du niveau d'éducation et une augmentation de l'espérance de vie, les défis en termes de pauvreté et d'inégalités restent très importants. L'analyse du questionnaire des indicateurs de base du bien-être (QUIBB) 2015 révèle un profil de pauvreté évoluant d'une pauvreté rurale à une pauvreté urbaine. La croissance n'a pas été inclusive. L'incidence de la pauvreté est plus importante dans les ménages gérés par des femmes. Les inégalités de revenus (mesurées par le coefficient de Gini) ont empiré, passant de 0.36 en 2006 à 0.38 en 2015. Le Togo est aujourd'hui dans une phase cruciale de sa politique, il élabore en effet son prochain Plan national de développement, lequel fait suite à la SCAPE 2013-17.

Le Togo reconnait la protection sociale comme un droit pour tout individu et une condition du progrès économique et social en adhérant à la plupart des conventions et accords internationaux y relatifs, notamment la Convention 102 de l'Organisation internationale du travail (OIT), qui définit un niveau minimum de sécurité sociale pour les populations, et la Convention multilatérale de la sécurité sociale instituée par la Conférence interafricaine de la prévoyance sociale (Cipres).

Il est important d'évaluer la situation actuelle des politiques fiscales et des dépenses sociales afin d'optimiser les dépenses publiques, et ainsi promouvoir de meilleures conditions de vie et une société inclusive au Togo. En effet, il est nécessaire de prendre en compte aussi bien les dépenses que les sources de revenus utilisées pour financer ces dépenses afin d'assurer leur pérennité et leur adéquation d'un point de vue redistributif. De plus, prendre en compte ces deux aspects permet d'identifier un effet «net» de la politique sociale, qui reflète les niveaux de revenu des ménages après imposition et après réception des transferts gouvernementaux.

Cette étude utilise une méthodologie reconnue internationalement, développée par l'Institut Commitment to Equity (CEQ). Elle estime l'impact redistributif des revenus fiscaux (les impôts) et des dépenses fiscales à travers les transferts monétaires, non monétaires, et les subventions, sur les revenus des ménages et les inégalités. Ce type de méthodologie a également été utilisé par l'OCDE, notamment par Causa et Hermansen $\left(2017_{[1]}\right)$ et dans le cadre de la Base de données sur la distribution des revenus ${ }^{1}$.

Deux conclusions ressortent de cette analyse : la politique fiscale réduit les inégalités au Togo, mais appauvrit les ménages les plus pauvres.

Le coefficient de Gini, estimé sur la base des revenus qui n'incluent ni les impôts (directs et indirects) ni les subventions ou transferts non monétaires (le revenu dit « de marché »),

${ }^{1}$ http://www.oecd.org/fr/social/donnees-distribution-revenus.htm 
atteint 0.405 , soit 5.2 points de pourcentage de plus que le coefficient de Gini estimé sur la base des revenus qui incluent ces éléments de politique fiscale (le revenu dit «final»), qui est de 0.38 .

L’impact des transferts sur la pauvreté est au contraire négatif pour la plupart des ménages pauvres, qui deviennent des payeurs nets dans le système fiscal. Les subventions en électricité et dans l'agriculture reçues par les ménages les plus pauvres sont en effet moins importantes que la somme totale payée par ces ménages en impôts indirects. La politique fiscale togolaise augmente ainsi le taux de pauvreté de 6 points - ce qui correspond à la moyenne des pays de comparaison. Les subventions reçues par les ménages pauvres et vulnérables sont plus faibles que la TVA payée, ils sont donc des payeurs nets dans le système fiscal togolais, contribuant plus aux recettes publiques qu'ils ne bénéficient des dépenses publiques. Le Togo n'a pas à ce jour de programme de transferts monétaires de taille suffisante (il couvre moins de $0.5 \%$ de la population) pour protéger les ménages les plus vulnérables.

Le Togo est un cas particulier dans la mesure où l'impact fiscal est quasiment entièrement le résultat des politiques d'accès à la santé et à l'éducation publique. La plupart des systèmes fiscaux réduisent les inégalités à travers des transferts directs ou des subventions, ainsi que les services publics. Les transferts directs et les subventions n'ont cependant pas d'impact sur les inégalités au Togo. 


\section{Table des matières}

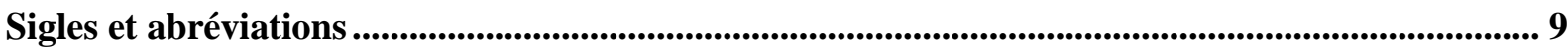

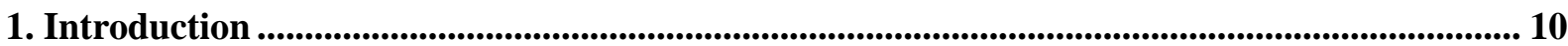

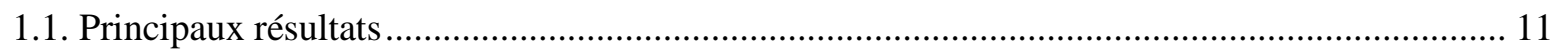

2. Dépenses sociales et recettes au Togo ................................................................................................................ 11

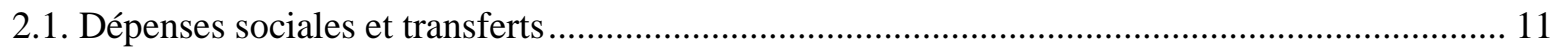

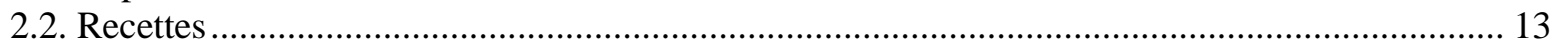

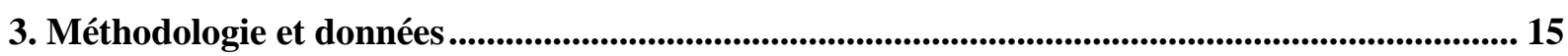

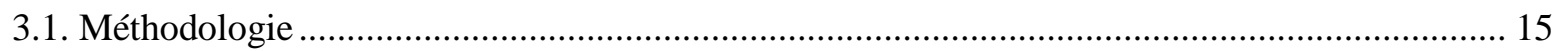

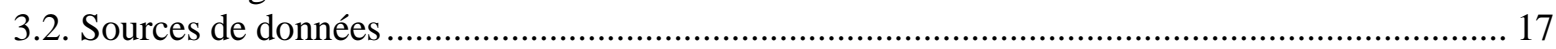

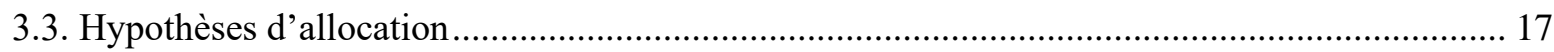

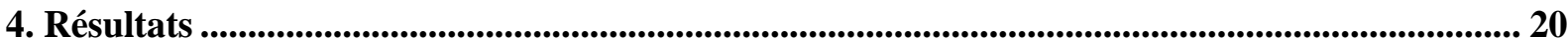

4.1. Quel est l'effet de la politique fiscale sur les inégalités et la pauvreté ?................................... 20

4.2. Les éléments de politique fiscale sont-ils en faveur des plus pauvres? Sont-ils progressifs ou

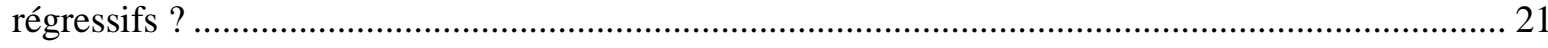

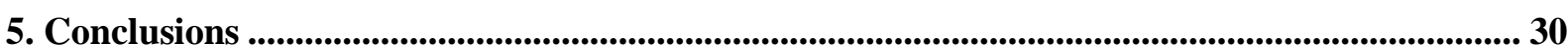

5.1. La politique fiscale réduit les inégalités.......................................................................... 30

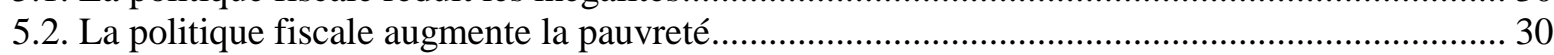

5.3. Limites de la méthodologie : les transferts non monétaires à travers les services sont importants........ 31

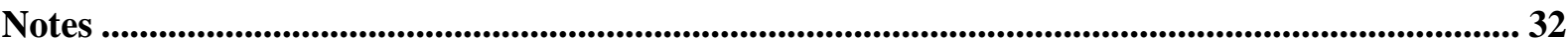

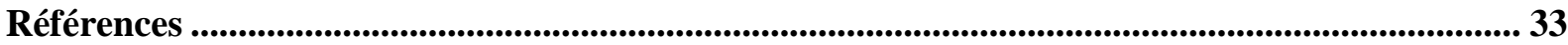

\section{Tableaux}

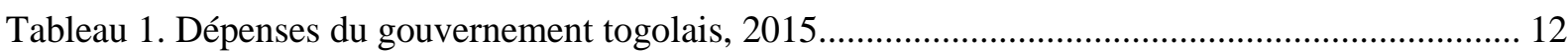

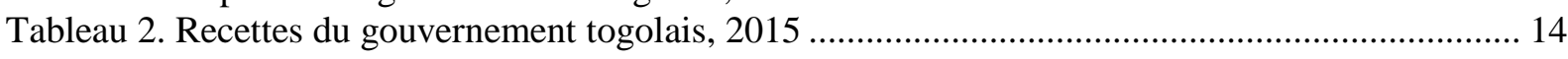

\section{Graphiques}

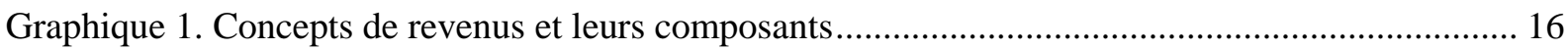

Graphique 2. La politique fiscale togolaise réduit les inégalités mais augmente la pauvreté ............... 20

Graphique 3. Incidence des subventions en électricité et intrants agricoles au Togo ........................... 22

Graphique 4. Taux de pauvreté sur la base des revenus pré- et post-fiscaux dans 11 pays africains .... 23

Graphique 5. Taux de scolarisation (dans la population générale) au Togo par niveau de scolarité et décile 
Graphique 6. Courbe de Lorenz (revenu disponible) et courbes de concentration des allocations pour chaque niveau d'éducation au Togo

Graphique 7. Variation et moyenne du coefficient d'efficacité d'impact par instrument de politique fiscale

Graphique 8. Fréquence de déclaration d'un incident de santé et utilisation du système de santé au Togo.

Graphique 9. Courbe de Lorenz (revenu disponible) et courbes de concentration des bénéfices de santé (rang=revenu disponible) au Togo

Graphique 10. Incidence des transferts, subventions, transferts non monétaires et TVA .....................29

Graphique 11. Contributions marginales aux inégalités de revenu, par instrument de politique fiscale ...... 30 


\section{Sigles et abréviations}

$\begin{array}{ll}\text { AgriPME } & \text { Porte-monnaie électronique pour les agriculteurs togolais } \\ \text { CEQ } & \text { Commitment to Equity } \\ \text { FCFA } & \text { Francs de la Communauté financière d'Afrique } \\ \text { INAM } & \text { Institut national d'assurance maladie } \\ \text { OIT } & \text { Organisation internationale du travail } \\ \text { PIB } & \text { Produit intérieur brut } \\ \text { QUIBB } & \text { Questionnaire des indicateurs de base du bien-être } \\ \text { SCAPE } & \text { Stratégie de croissance accélérée et de promotion de l'emploi } \\ \text { TVA } & \text { Taxe sur la valeur ajoutée }\end{array}$




\section{Introduction}

Le gouvernement togolais a entrepris des réformes importantes après la crise des années 90 dans le but d'améliorer les conditions de vie de la population grâce à des politiques publiques adaptées. Le pays a notamment adopté en 2006 une stratégie nationale de développement de long terme sur la base des Objectifs du Millénaire pour le développement et, plus récemment, la Stratégie de croissance accélérée et de promotion de l'emploi 2013-17 (SCAPE). La croissance économique est stable à environ $5 \%$ grâce à une amélioration de la productivité agricole et d'importants investissements dans les infrastructures publiques.

Malgré des améliorations du niveau d'éducation et une augmentation de l'espérance de vie, les défis en termes de pauvreté et d'inégalités restent très importants. Le taux d'alphabétisation des jeunes entre 15 et 24 ans a atteint $83.5 \%$ en 2015, et l'espérance de vie moyenne des Togolais est de 59.9 ans. Cependant, le score du Togo sur l'index de développement humain ( 0.487 en 2015), bien qu'au-dessus de la moyenne de ses voisins de l'Union économique et monétaire ouest-africaine, et malgré une augmentation depuis 2005 (0.436), reste loin de la moyenne des pays d'Afrique subsaharienne.

L'analyse du questionnaire des indicateurs de base du bien-être (QUIBB) 2015 révèle un profil de pauvreté évoluant d'une pauvreté rurale à une pauvreté urbaine. Le taux de pauvreté moyen a baissé, passant de $58.7 \%$ en 2011 à $55.1 \%$ en 2015, avec une incidence de la pauvreté beaucoup plus élevée en zones rurales (69 \%) qu'en zones urbaines. Cependant, la pauvreté urbaine a augmenté de façon importante à Lomé et dans sa périphérie, passant de $28.5 \%$ en 2011 à $34.8 \%$ en 2015 ; l'extrême pauvreté a également progressé de $9.1 \%$ en 2011 à $12.9 \%$ en 2015. L'incidence de la pauvreté est plus importante dans les ménages gérés par des femmes. Les inégalités de revenus (mesurées par le coefficient de Gini) ont empiré, passant de 0.36 en 2006 à 0.38 en 2015. La croissance n'a pas été inclusive.

Le Togo est aujourd'hui dans une phase cruciale de sa politique, il élabore en effet son prochain Plan national de développement, lequel fait suite à la SCAPE 2013-17. Le gouvernement s'était engagé dans le cadre de la SCAPE à promouvoir une croissance inclusive, et le Président Faure Gnassingbé a souligné cette priorité en déclarant son troisième quinquennat (2015-20) comme celui du mandat social. Le Togo reconnaît la protection sociale comme un droit pour tout individu et une condition du progrès économique et social en adhérant à la plupart des conventions et accords internationaux y relatifs, notamment la Convention 102 de l'Organisation internationale du travail, qui définit un niveau minimum de sécurité sociale pour les populations, et la Convention multilatérale de la sécurité sociale instituée par la Conférence interafricaine de la prévoyance sociale.

Il est important d'évaluer la situation actuelle des politiques fiscales et des dépenses sociales afin d'optimiser les dépenses publiques, et ainsi promouvoir de meilleures conditions de vie et une société inclusive au Togo. En effet, il est nécessaire de prendre en compte aussi bien les dépenses que les sources de revenus utilisées pour financer ces dépenses afin d'assurer leur pérennité et leur adéquation d'un point de vue redistributif. De plus, prendre en compte ces deux aspects permet d'identifier un effet «net» de la politique sociale, qui reflète les niveaux de revenu des ménages après imposition et après réception des transferts gouvernementaux.

Cette étude utilise une méthodologie reconnue internationalement, développée par l'institut Commitment to Equity (CEQ). Elle estime l'impact redistributif des revenus fiscaux (les impôts) et des dépenses fiscales à travers les transferts monétaires, non monétaires, et les 
subventions, sur les revenus des ménages et les inégalités. Cette méthode a été utilisée notamment en Amérique latine, en Afrique, en Europe et en Asie, permettant de comparer les résultats du Togo avec d'autres pays.

Les inégalités de revenu résultent de divers facteurs, notamment l'accès aux soins ou à l'éducation, aux infrastructures ou à l'électricité. Une partie importante de ces facteurs peut être influencée par la politique fiscale. Cette étude se concentre sur l'année fiscale 2015, celle-ci coïncidant avec les données collectées à travers le QUIBB et l'étude sur l'Outil d'options de politiques de protection sociale menée en 2017-2018 (EUSPS et BIT, 2018[1]).

\subsection{Principaux résultats}

La politique fiscale réduit les inégalités au Togo, mais appauvrit les ménages les plus pauvres. Les inégalités diminuent en prenant en compte les transferts non monétaires et les subventions, tout en soustrayant les impôts indirects (principalement la taxe sur la valeur ajoutée [TVA]) dans les revenus des ménages. Le coefficient de Gini est ainsi réduit de 5.2 points suite à ces transferts. Cet impact est supérieur à la moyenne pour un pays de la taille du Togo : dans $11^{1}$ pays aux revenus faibles et moyens en Afrique entre 2010 à 2015 les politiques fiscales ont réduit les inégalités de 7.5 points de Gini. En excluant trois pays à revenus moyens (Namibie, Afrique du Sud et Zambie), cette moyenne est de 3.3 points.

L'impact des transferts sur la pauvreté est au contraire négatif pour la plupart des ménages pauvres, qui deviennent des payeurs nets dans le système fiscal. Les subventions en électricité et dans l'agriculture reçues par les ménages les plus pauvres sont en effet moins importantes que la somme totale payée par ces ménages en impôts indirects. La politique fiscale togolaise augmente ainsi le taux de pauvreté de 6 points de pourcentage, ce qui correspond à la moyenne des pays de comparaison.

Le Togo est un cas particulier dans la mesure où l'impact fiscal est quasiment entièrement le résultat des politiques d'accès à la santé et à l'éducation publique. La plupart des systèmes fiscaux réduisent les inégalités à travers des transferts directs ou des subventions, ainsi que les services publics. Les politiques fiscales à travers les transferts directs et subventions au Togo n'ont cependant pas d'impact sur les inégalités.

Ce rapport est organisé en cinq sections. La deuxième section donne une vue d'ensemble des transferts les plus importants et des impôts au Togo. La troisième section décrit la méthodologie et les sources de données. Les résultats, ainsi qu'une comparaison de ces résultats avec ceux d'autres pays, sont présentés dans la quatrième section. La dernière section conclut sur les implications en termes de politiques publiques au Togo.

\section{Dépenses sociales et recettes au Togo}

\subsection{Dépenses sociales et transferts}

Les dépenses sociales au Togo peuvent être divisées en trois catégories : les transferts non monétaires, les transferts directs et les subventions indirectes. 
Les dépenses fiscales pour l'année fiscale 2015 sont présentées dans le Tableau 1. Les dépenses pour la défense et la sécurité ne sont pas couvertes par l'analyse, qui prend cependant en compte l'essentiel du portefeuille de dépenses sociales.

Tableau 1. Dépenses du gouvernement togolais, 2015

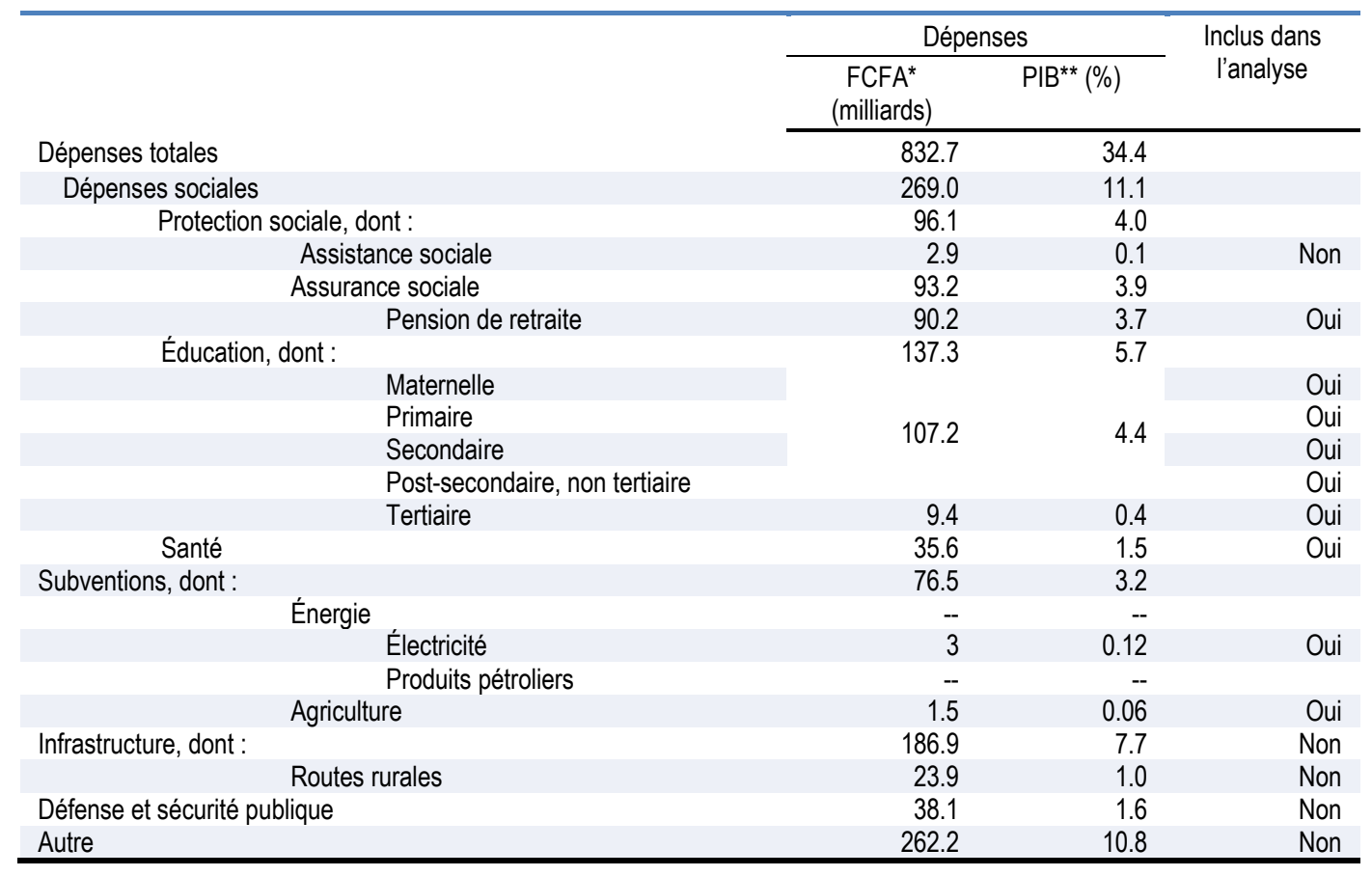

Notes: *Francs de la Communauté financière d'Afrique (FCFA). **Produit intérieur brut (PIB). Les dépenses et revenus dans cette étude ne peuvent pas toujours être reliés au QUIBB pour diverses raisons présentées dans la section 3.

Sources: (BIT, à paraître ${ }_{[2]}$; FMI, 2017 [3]).

\subsubsection{Transferts non monétaires}

\section{Éducation}

L'éducation est obligatoire au Togo pour les enfants entre 6 et 15 ans. Le taux de scolarisation net en 2014 était de $91.2 \%$ (94.3\% pour les garçons et $88 \%$ pour les filles). Malgré la mise en place de la gratuité des frais de scolarité en 2008, l'accès à l'éducation reste problématique, en particulier pour les enfants les plus défavorisés. En 2016, environ 1.5 million d'enfants ont bénéficié d'une exemption des frais de scolarité dans le primaire, représentant une moyenne de 1225 FCFA (francs de la Communauté financière d'Afrique) par enfant. D'autres programmes ont été mis en place pour soutenir la scolarisation des enfants, notamment à travers les cantines scolaires et les dons de fournitures scolaires. Ces programmes ne touchent cependant qu'une petite partie des enfants : en 2016, seuls $5 \%$ des enfants en âge d'être scolarisés ont reçu des repas gratuits et moins de 7300 enfants ont reçu des fournitures scolaires (EUSPS et BIT, 2018 ${ }_{[1]}$ ). Quant au programme de subvention pour les filles, il n'a concerné que 87000 filles en 2015.

\section{Santé}

Un régime obligatoire d'assurance maladie pour les agents publics et assimilés a été mis en place en 2011, et le volet protection sociale de la SCAPE 2013-17 inclue un axe dédié à la 
protection sociale mentionne la mise en place progressive d'un système de couverture santé universelle. L'Institut national d'assurance maladie (INAM) est l'organisme de gestion principal de ce régime et l'acteur principal dans l'extension de la couverture maladie, notamment aux travailleurs formels du secteur privé, aux travailleurs informels, aux travailleurs agricoles et aux personnes vulnérables. En 2016, seule $3.23 \%$ de la population en âge actif (18 à 60 ans) était couverte par l'assurance maladie obligatoire en qualité d'assurée principale de l'INAM (EUSPS et BIT, 2018 $\left.{ }_{[1]}\right)$.

\subsubsection{Subventions indirectes}

\section{Électricité}

La proportion des ménages utilisant l'électricité est passée de $39.2 \%$ en 2011 à $48.3 \%$ en 2015 (INSEED, 2015[4] ). Bien qu'ayant augmenté entre 2011 et 2015, le taux d'accès à l'électricité révèle des inégalités importantes entre les zones rurales et les zones urbaines : il atteignait $79 \%$ en 2015 dans les zones urbaines, contre $12 \%$ en zones rurales $(12 \%){ }^{2}$ Le programme de subvention au Togo met à la disposition des producteurs d'électricité des facteurs de production subventionnés.

\section{Subventions pour l'agriculture}

Les réformes récentes des subventions à l'agriculture (e.g. AgriPME) ont pour but d'améliorer la distribution d'engrais aux producteurs agricoles à travers l'achat de produits subventionnés dans des centres de distribution privés. Cette subvention est accordée aux producteurs vulnérables qui peuvent, s'ils sont identifiés en amont, s'enregistrer aux ventes d'engrais en présentant leur carte d'identité ainsi qu'un numéro de téléphone valide (lié aux opérateurs Moov ou TogoCel). Les bénéficiaires doivent être résidents de leur village depuis au moins trois ans et avoir une surface cultivable entre 0.25 et 1 hectare pour le maïs, le riz ou le sorgho. Une fois les agriculteurs éligibles recensés, les fonds de subventions du gouvernement sont convertis par les opérateurs téléphoniques en monnaie électronique. Ces subventions ont atteint 1.5 milliard FCFA en 2015.

\subsection{Recettes}

Les principales sources de recettes publiques en 2015 sont présentées dans le Tableau 2. Avec des recettes fiscales totales représentant $21.3 \%$ du produit intérieur brut (PIB), ces recettes représentent environ les deux tiers de tous les revenus et dons. En comparaison, ce taux atteint $16.1 \%$ au Cameroun et au Rwanda, $17.8 \%$ en Côte d'Ivoire, et la moyenne dans les pays de l'OCDE est de $34.4 \%$. Depuis l'an 2000, le Togo a réussi à augmenter de 10 points de pourcentage son ratio de recettes fiscales par rapport au PIB, avec une augmentation importante des recettes fiscales due aux améliorations et au renforcement de la structure institutionnelle, notamment entre la Direction générale des impôts, la Direction générale des douanes et la Direction générale du trésor et de la comptabilité publique. Ainsi, le gouvernement togolais a lancé une réforme importante afin d'augmenter les revenus domestiques, créant notamment l'Office togolais des recettes en 2012.

Les dons, qui représentent entre 2 et $3 \%$ du PIB, sont équivalents en taille aux recettes non fiscales.

Les impôts directs représentent $4 \%$ du PIB en recettes fiscales. Les impôts personnels et de revenus sont inclus dans l'analyse. Les impôts sur les entreprises ne sont cependant pas inclus, étant donné la difficulté à allouer ces impôts aux individus et ménages du QUIBB. 
Les impôts sur la propriété ne sont pas inclus non plus étant donné le manque d'informations dans le QUIBB à ce sujet.

Les impôts indirects, composés de la TVA et des droits de douane, représentent $17 \%$ du PIB en 2015, soit plus de quatre fois le montant des impôts directs. La TVA représente presque $90 \%$ de tous les impôts indirects et est allouée aux ménages selon leur consommation de biens et services couverts par le système de TVA dans cette analyse. Les droits de douanes ne sont pas pris en compte en raison du manque d'informations sur la consommation de biens importés.

Tableau 2. Recettes du gouvernement togolais, 2015

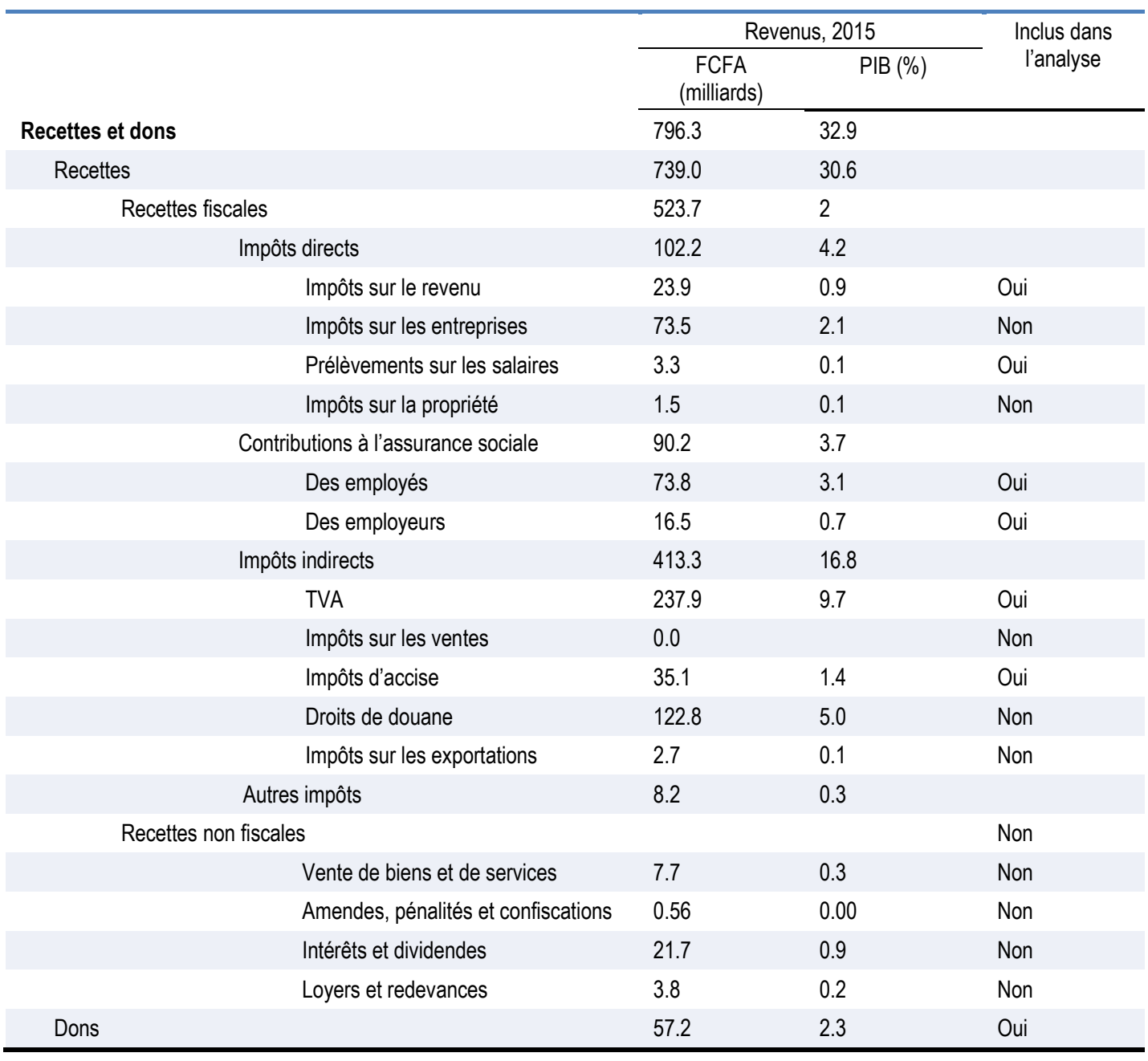

Note : Les dépenses et revenus dans cette étude ne peuvent pas toujours être reliés au QUIBB pour diverses raisons présentées dans la section 3 .

Source: OCDE/ATAF/CUA (2017 $[5])$, Statistiques des recettes publiques en Afrique 2017, http://dx.doi.org/10.1787/9789264280854-en-fr. 


\section{Méthodologie et données}

\subsection{Méthodologie}

Cette étude prend en compte des aspects spécifiques de la politique fiscale, des programmes, dépenses et recettes décrits dans la section précédente, et les alloue aux individus et ménages dans les enquêtes selon leurs caractéristiques. Cette méthodologie permet d'identifier au niveau micro les individus bénéficiant des dépenses ou, à l'inverse, contribuant aux recettes. Cette allocation établie, différentes mesures de la pauvreté et de l'appauvrissement, de l'inégalité et de la progressivité sont calculées. Ces mesures permettent d'identifier la redistribution accomplie à travers différents concepts de revenus des ménages, notamment pré-fiscal et post-fiscal (Graphique 1). 


\section{Graphique 1. Concepts de revenus et leurs composants}

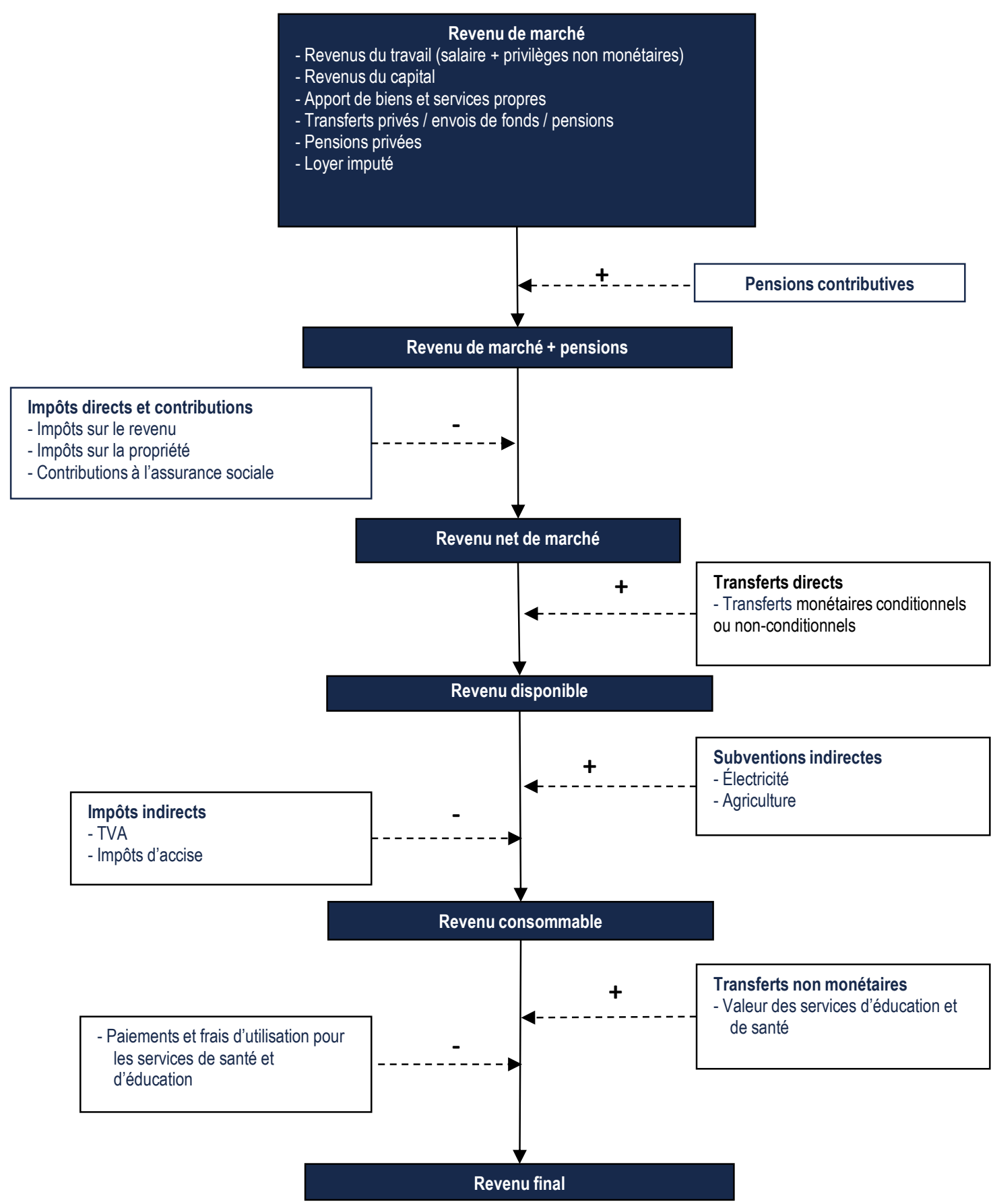

Sources: Lustig, N. (ed.) (2016[6]), Commitment to Equity Handbook: Estimating the Impact of Fiscal Policy on Inequality and Poverty, www.commitmentoequity.org/publications/handbook.php.

Le QUIBB ne contient pas assez d'informations pour calculer le revenu de marché. Cette analyse se concentre donc sur les dépenses de consommation comme mesure principale des revenus. L'analyse repose sur l'hypothèse que les dépenses totales de consommation - 
incluant la valeur du loyer imputée ainsi que la valeur de l'autoproduction - correspondent au concept de revenu disponible.

L'analyse n'incluant pas les transferts directs (ces derniers ne bénéficiant qu'à un nombre restreint de personnes $)^{3}$, il n'existe pas de différences entre le revenu disponible et le revenu net de marché.

\subsection{Sources de données}

La base de données principale pour cette analyse aux niveaux individuel et ménage est le QUIBB 2015. Cette enquête contient des informations sur les transferts non monétaires, ainsi que sur les niveaux de consommation qui permettent d'imputer l'imposition effective indirecte, ainsi que des montants des transferts et subventions effectifs.

La principale source de données pour les recettes du gouvernement est la publication Statistiques des recettes publiques en Afrique (OCDE/ATAF/CUA, 2017 ${ }_{[5]}$ ). Afin de pouvoir imputer des taux effectifs de collecte de recettes, qui diffèrent souvent du taux statutaire, nous réduisons proportionnellement le montant collecté à travers la TVA dans les ménages du QUIBB de façon à ce que le ratio recettes de la TVA [provenant de (OCDE/ATAF/CUA, $\left.2017_{[5]}\right)$ / dépenses de consommation privées dans les comptes nationaux aux Togo soit équivalent au ratio recettes de la TVA des ménages du QUIBB / valeur cumulée des dépenses de consommation des ménages dans le QUIBB.

Cependant, certaines transactions sont exemptées de TVA : la nourriture, les biens agricoles ou encore les services de santé ou d'éducation. Les biens exemptés de TVA ont généralement un taux implicite d'imposition plus faible car les producteurs de biens exemptés n'appliquent pas de TVA sur la vente finale. De plus, certaines transactions non exemptées pourraient être effectuées à un taux moindre que le taux statutaire en raison de l'évasion fiscale, de la faiblesse de l'administration fiscale, ou parce que les deux parties engagées dans la transaction ne sont pas enregistrées dans le système de TVA. La collecte de la TVA, par exemple telle que reportée dans les budgets de l'État, sera ainsi moindre que le maximum possible (à un niveau de transactions donné) à cause des biens exemptés ainsi que pour les raisons mentionnées ci-dessus.

Nous ne pouvons pas désagréger les sommes de TVA indiquées par rapport aux dépenses totales de ménages dans les comptes nationaux. Nous choisissons ici d'utiliser un taux implicite de TVA sur les transactions exemptées, tel que la somme de 1) la valeur totale des biens exemptés dans l'enquête ménage multipliée par la valeur implicite du taux de TVA sur ces transactions exemptées ; et de 2) la valeur totale des transactions standards dans l'enquête ménage multipliée par le taux explicite de TVA pour les transactions standard, donne un taux de TVA équivalent au taux de TVA décrit ci-dessus.

\subsection{Hypothèses d'allocation}

Les éléments de politiques fiscales sont alloués sur la base d'observations directes des individus et ménages dans la mesure du possible. Par exemple, si un individu déclare dans une enquête avoir payé une certaine somme en TVA, cette donnée est considérée comme observée et présumée correspondre aux données administratives budgétaires de l'année.

$\mathrm{Au}$ Togo, peu d'éléments de la politique fiscale peuvent être alloués à travers ces observations directes. La section ci-dessous décrit la méthodologie utilisée pour pallier ce manque de données. 


\subsubsection{Impôts directs et pensions : simulations}

Les impôts directs - impôt sur le revenu et taxe sur les cotisations salariales - ne sont pas observés directement dans le QUIBB 2015. Nous créons un sous-échantillon d'individus qui déclarent avoir travaillé dans un poste permanent ou sous contrat durant les sept jours précédents, et qui reçoivent une compensation non-salariale, afin de simuler les impôts directs. Nous appliquons ensuite les règles suivantes : si un individu reçoit plus de la moitié de sa compensation à travers une compensation non-salariale, il est éligible à l'impôt sur le revenu. S'il reçoit moins de la moitié de sa compensation à travers une compensation nonsalariale, il est considéré comme éligible aux cotisations salariales. Une fois cet échantillon établi, nous créons un groupe de payeurs d'impôts en identifiant les individus correspondant aux déciles sept à dix (c'est-à-dire les plus riches) de la distribution de consommation par tête parmi ces payeurs éligibles. Nous allouons ensuite à chaque individu correspondant à cet échantillon une proportion des impôts totaux équivalente à la proportion de revenu cumulé (dans l'échantillon de payeurs d'impôts) de cet individu.

Par ailleurs, les contributions au système de retraite des agents de la fonction publique ne sont pas déclarées directement dans le QUIBB. Nous suivons une méthodologie similaire à celle décrite ci-dessus pour allouer les contributions au système de pensions. Nous sélectionnons d'abord un sous-échantillon d'individus ayant un contrat permanent d'agent de la fonction publique. Nous sélectionnons ensuite parmi ces individus ceux qui déclarent une compensation non-salariale. Sont alors considérés comme contributeurs les individus employés dans la fonction publique et recevant une compensation non-salariale. L'allocation d'une proportion des contributions de pension totale a lieu sur la base de la proportion des revenus (au sein du groupe de contributeurs potentiels) représentée par un individu.

\subsubsection{TVA : sur la base des dépenses déclarées}

Nous ne pouvons pas identifier directement les paiements de TVA dans le QUIBB. Nous calculons, pour chaque dépense, la proportion de la valeur de cette dépense correspondant soit à la TVA explicite (pour les biens et services standards), soit à la TVA implicite (pour les biens exemptés, plus coûteux en raison de la TVA payée sur les facteurs de production). Nous échelonnons ces taxes sur deux bases afin de déterminer la proportion des dépenses correspondant à la TVA. Le premier facteur d'échelonnement est créé en comparant la TVA totale dans les recettes des comptes nationaux avec la consommation des ménages privés dans les comptes nationaux. Ce ratio indique la proportion de TVA « effective », c'est-à-dire la TVA collectée par rapport à la valeur de vente (incluant la TVA) de la base imposable. À partir de cette proportion nous déterminons le niveau d'imposition qui, lorsqu'il est appliqué à la valeur nette de la TVA, résulterait dans la valeur des ventes enregistrée dans les comptes nationaux. Nous choisissons ensuite une proportion à travers laquelle le taux effectif sur les biens exemptés est plus faible que le taux effectif sur les biens standards. Par exemple, au Togo nous estimons un taux effectif pour les biens exemptés représentant $65 \%$ du taux effectif pour les biens standards.

Une fois le taux global effectif et la proportion des biens exemptés identifiés (par rapport à la consommation totale) dans l'enquête ménage, et après avoir identifié le taux effectif pour les biens exemptés (par rapport au taux effectif pour les biens standards), nous pouvons calculer le taux effectif sur les biens standards. Nous appliquons ensuite ces taux de TVA sur toutes les dépenses de consommation, pour chaque bien exempté et chaque bien standard, afin d'estimer les dépenses de TVA totales par ménage.

Nous excluons explicitement les achats informels, qui ne sont pas inclus dans les ventes correspondant à une taxation indirecte. Cependant, étant donné que nous ne prenons pas en 
compte les achats informels, le manque à gagner d'impôts collectés (et donc alloués dans le QUIBB) dû à ces achats informels ou une faible collecte des impôts est alloué à tous les ménages effectuant des dépenses soumises à la TVA.

\subsubsection{Subventions d'électricité}

Les tarifs d'électricité ne sont pas subventionnés directement, les producteurs d'électricité bénéficient d'une subvention de leurs facteurs de production. Suivant l'hypothèse que ces subventions sont transmises aux consommateurs, nous partons du principe que ces subventions sont linéaires avec l'utilisation et que les utilisateurs de plus gros volume d'électricité bénéficient du plus gros volume de subventions. Quant à la TVA, nous divisons le total des subventions allouées afin que le ratio de subventions allouées dans l'enquête QUIBB par rapport aux dépenses des ménages dans l'enquête soit égal au ratio de subventions dans le budget par rapport à la consommation des ménages dans les comptes nationaux.

\subsubsection{Subventions des intrants agricoles}

Le programme AgriPME concerne les ménages agricoles qui possèdent ou louent un terrain (mais ne sont pas métayers), et qui possèdent un téléphone mobile et ont effectué des dépenses de téléphonie mobile dans l'année précédente. La subvention est allouée sur la base du volume, prenant ainsi la dimension de la taille des dépenses en engrais - mesurée par la proportion de terrains que le ménage possède par rapport à la somme totale de terrains possédés par les bénéficiaires de la subvention - ainsi que la proportion de revenu des ménages par rapport à la somme totale à travers tous les bénéficiaires de la subvention.

Le montant total des subventions allouées dans le QUIBB est mis à l'échelle des budgets totaux en appliquant le ratio de la consommation privée des ménages déclarée dans les comptes nationaux aux consommation privées des ménages dans le QUIBB.

\subsubsection{Transferts non monétaires}

Les dépenses publiques en éducation et santé sont allouées aux ménages du QUIBB comptant au moins un membre utilisant le système public d'éducation ou le système de santé public. Les dépenses en transferts non monétaires sont divisées par le nombre total d'utilisateurs dans le QUIBB afin d'obtenir une subvention par élève ou par patient - cette subvention uniforme est alors appliquée à tous les utilisateurs identifiés directement. Ainsi, un ménage avec un enfant scolarisé dans le primaire, un enfant scolarisé au niveau secondaire, une visite dans un hôpital public et deux visites dans une clinique publique recevrait cinq subventions différentes correspondant aux cinq types de services utilisés. 


\section{Résultats}

\subsection{Quel est l'effet de la politique fiscale sur les inégalités et la pauvreté ?}

Les inégalités seraient plus importantes sans les éléments de politique fiscale pris en compte dans cette étude (voir Tableau 1 et Tableau 2), la politique fiscale réduit donc ces inégalités. Le coefficient de Gini, estimé sur la base des revenus qui n'incluent ni les impôts (directs et indirects) ni les subventions ou transferts non monétaires (le revenu dit « de marché »), atteint 0.405 , soit 5.2 points de pourcentage de plus que le coefficient de Gini estimé sur la base des revenus qui incluent ces éléments de politique fiscale (le revenu dit «final»), qui est de 0.350 (Graphique 2.A). Alors que les inégalités de revenus pré-fiscaux étaient marginalement plus élevées dans les zones urbaines que dans les zone rurales, ces dernières sont réduites de façon égalitaire entre les deux zones en conséquence de la politique fiscale.

\section{Graphique 2. La politique fiscale togolaise réduit les inégalités mais augmente la pauvreté}

A. Coefficient de Gini pour les revenus pré- et post-fiscaux

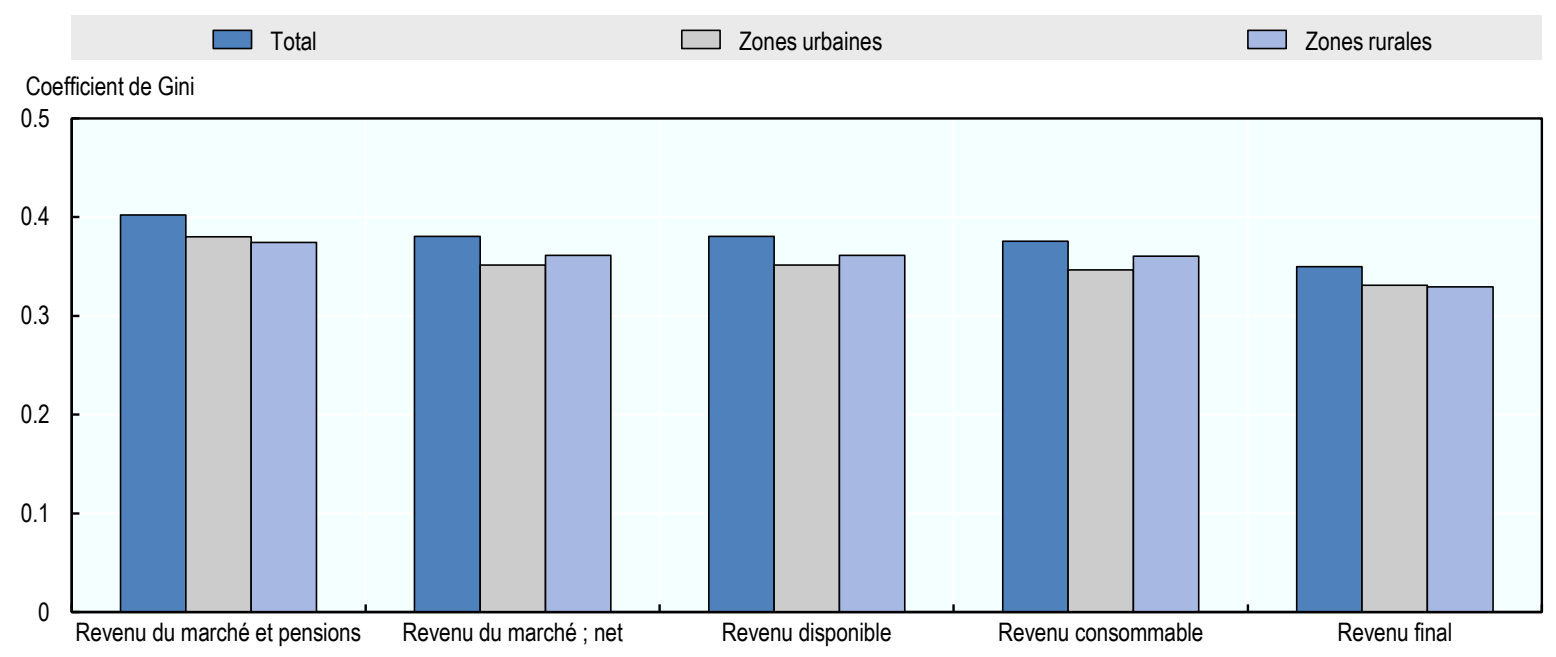

Sources: Calculs des auteurs sur la base du QUIBB 2015 (INSEED, 2015[4]). 
B. Taux de pauvreté dans les revenus pré- et post-fiscaux

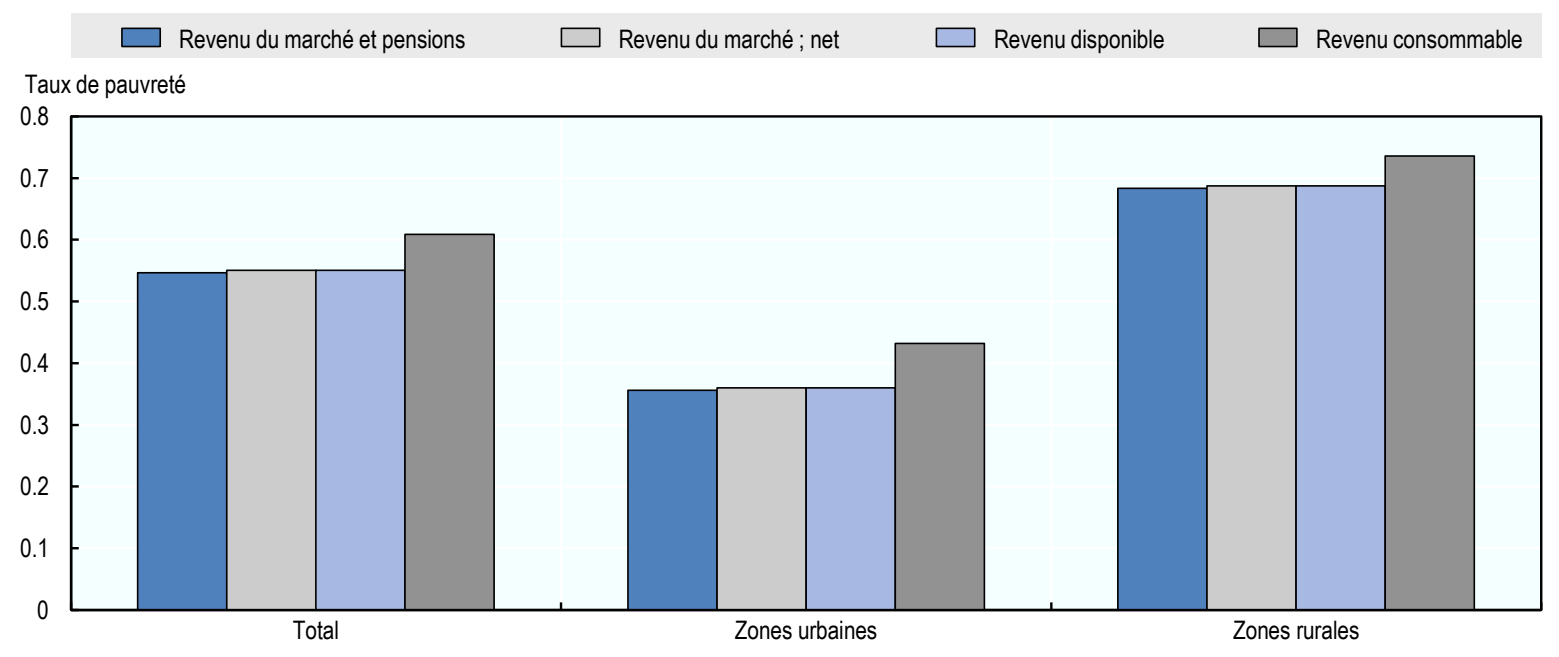

Sources: Calculs des auteurs sur la base du QUIBB 2015 (INSEED, 2015[4]).

Les impôts et les subventions augmentent la pauvreté (Graphique 2.B). Le taux de pauvreté (selon la ligne de pauvreté nationale) était par exemple de $55 \%$ sur la base des revenus disponibles, des revenus de marché net, ainsi qu'en prenant en compte les pensions. En ajoutant la valeur des subventions reçues (à travers l'achat d'engrais ou d'électricité) et en soustrayant la valeur des impôts directs et indirects (sur les revenus et sur la consommation), ce taux de pauvreté augmente la proportion de la population vivant sous le seuil de pauvreté, qui atteint $60 \%$ de la population totale. Bien que le taux de pauvreté soit plus élevé dans les zones rurales à la base, les politiques fiscales ont un effet moins appauvrissant dans ces dernières que dans les zones urbaines.

\subsection{Les éléments de politique fiscale sont-ils en faveur des plus pauvres ? Sont-ils progressifs ou régressifs?}

Les ménages qui consomment plus d'électricité reçoivent une part plus importante de la subvention en électricité, ces subventions sont donc régressives. En effet, la proportion de subventions reçues (par rapport à la somme totale de ces subventions) augmente avec le revenu disponible (Graphique 3). Cependant, ces mêmes subventions en électricité restent faibles en termes relatifs en comparaison avec le revenu des ménages : elles représentent moins de $0.25 \%$ du revenu (avant subventions) des ménages les plus riches. Étant donné qu'elles représentent une part encore moins importante du revenu des ménages les plus pauvres, il peut être conclu que ces subventions sont également régressives dans ce sens : les subventions en électricité reçues, en termes de pourcentage de revenu, augmentent avec le revenu. 
Graphique 3. Incidence des subventions en électricité et intrants agricoles au Togo

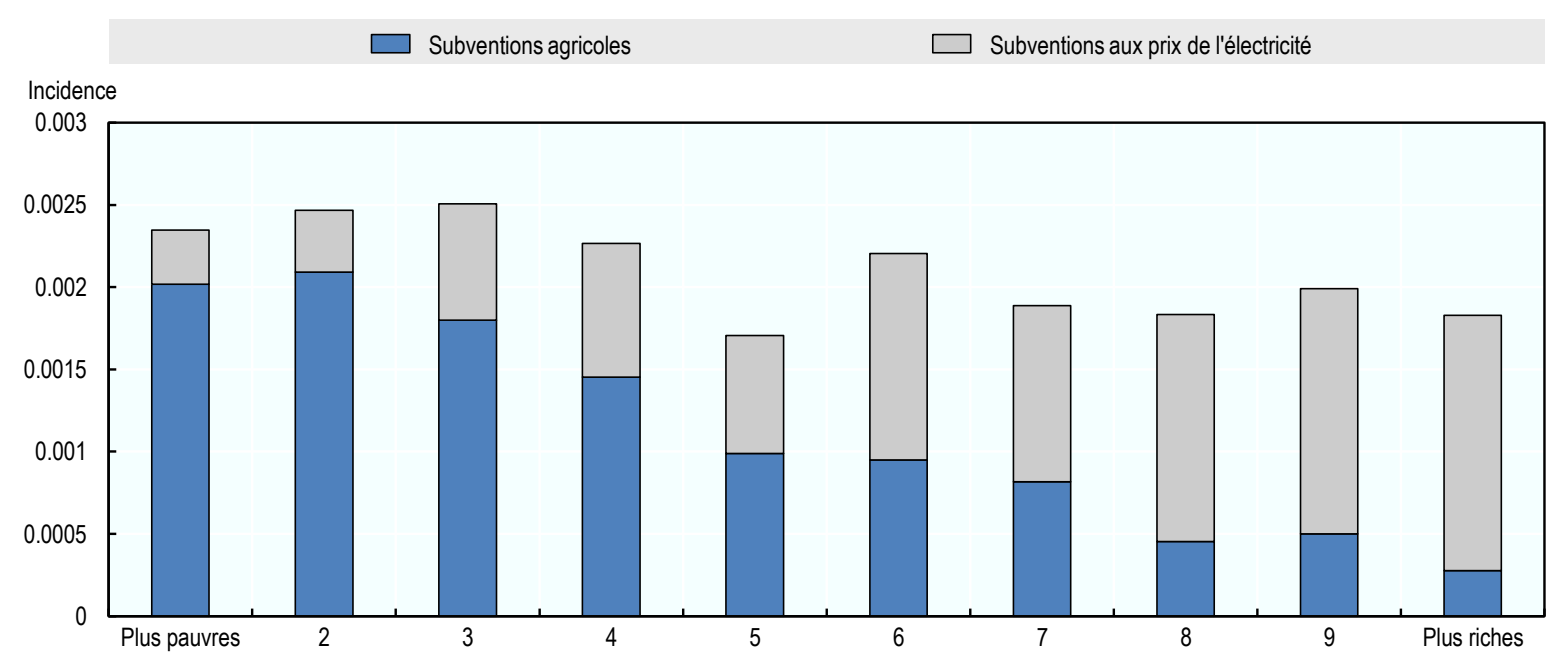

Sources: Calculs des auteurs sur la base du QUIBB 2015 (INSEED, 2015 $[4]$ ).

Le programme de subvention de l'engrais pour l'agriculture est quasiment neutre dans la mesure où, en moyenne, la moitié la plus pauvre des ménages reçoit $48 \%$ des subventions totales en agriculture. Ce même groupe ne reçoit que $10 \%$ des subventions en électricité. Le graphique 3 indique que les subventions totales (incluant l'électricité et l'agriculture) reçues par les ménages les plus pauvres représentent une proportion plus importante de leur revenu (avant subventions) que celle des ménages les plus riches. Les subventions totales représentent cependant en moyenne moins de $0.5 \%$ du revenu pour tous les ménages.

Les ménages les plus riches paient une partie plus importante des recettes totales collectées à travers la TVA et les taxes d'accises sur les produits pétroliers ${ }^{4}$ : le décile le plus riche paie environ $30 \%$ des recettes de TVA et $46 \%$ des taxes d'accises sur les produits pétroliers, alors que le décile le plus pauvre paie environ $2 \%$ des recettes de TVA et $0.1 \%$ des taxes d'accises sur les produits pétroliers. La TVA représente $10 \%$ du revenu des ménages les plus pauvres, alors qu'elle représente $12 \%$ du revenu des ménages les plus riches. La TVA est donc un élément de politique fiscale qui égalise la distribution des revenus au Togo. De même, les taxes d'accises sur les produits pétroliers représentent $0.1 \%$ des revenus dans le premier décile (qui consomme moins de pétrole) et $3.5 \%$ des revenus des plus riches.

Les subventions représentent moins de $1 \%$ des revenus (pour n'importe quel ménage à travers la distribution de revenus) et les impôts indirects représentent entre $11 \%$ et $15 \%$ des revenus; la position nette de tous les ménages après avoir ajouté ces deux éléments de politique fiscale est donc négative. Les ménages sont donc tous des payeurs nets dans le système fiscal, qui augmente ainsi le taux de pauvreté.

Ce résultat correspond à la moyenne des 11 pays à revenus faibles et moyen en Afrique subsaharienne pour les années fiscales 2010 et 2015 (Graphique 4). Seules l'Afrique du Sud et la Namibie, qui ont mis en place plusieurs transferts directs couvrant une partie importante de la population, ont un système fiscal qui favorise les ménages les plus pauvres, qui sont en situation nette positive. Dans les autres pays, le taux de pauvreté est inchangé, ou augmente en prenant en compte la politique fiscale. 


\section{Graphique 4. Taux de pauvreté sur la base des revenus pré- et post-fiscaux dans 11 pays africains}

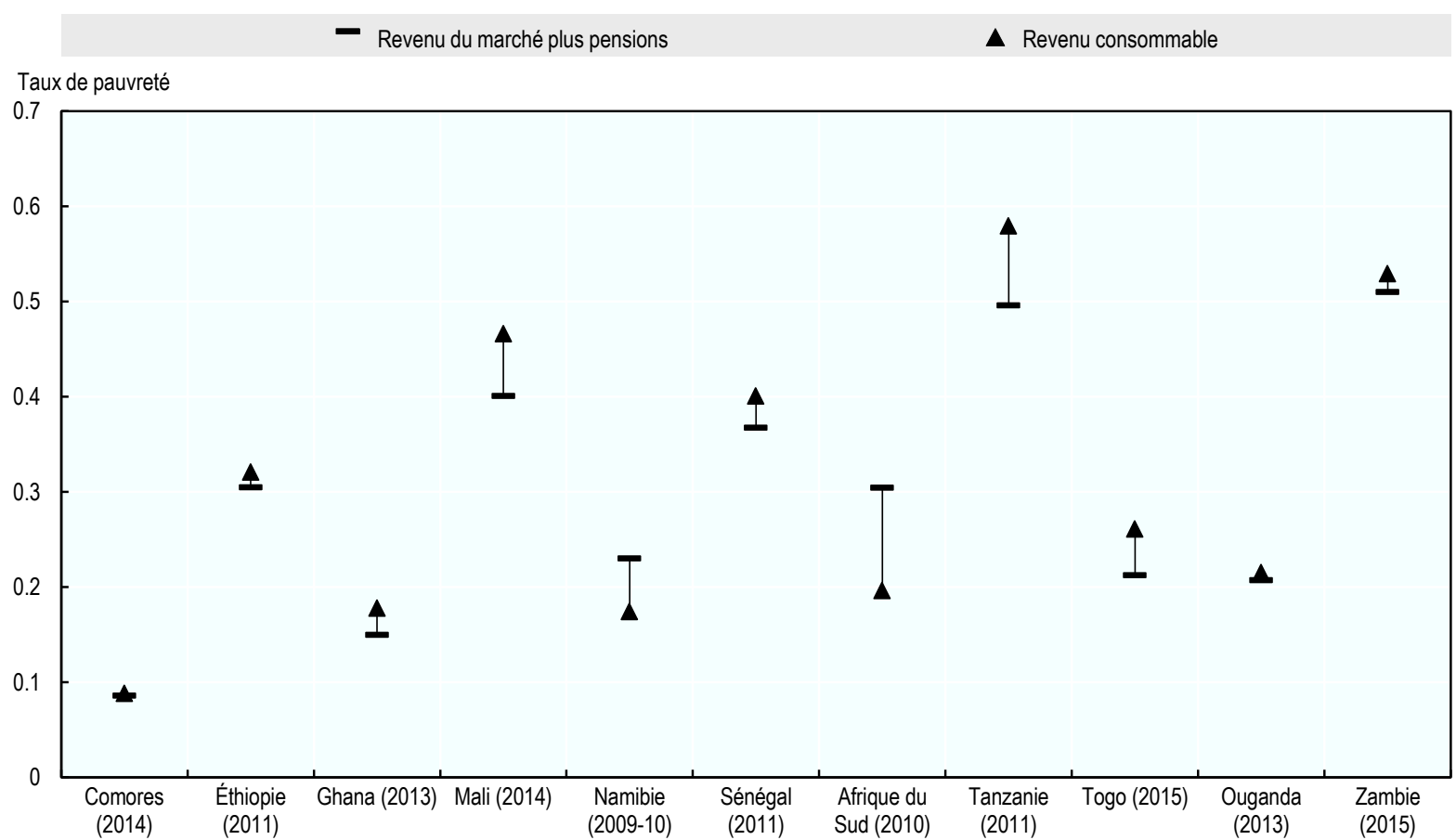

Notes: Pauvreté calculée sur la base de la ligne de pauvreté à 1.90 USD (dollar américain) par jour.

Sources: (de la Fuente, Jellema et Lustig, 2018 $[7]$ )

En 2015, les dépenses liées à l'éducation et aux soins de santé étaient environ 60 fois plus importantes que les dépenses de protection sociale (en excluant les pensions), environ deux fois plus importantes que les dépenses en subventions, et égales aux dépenses en infrastructure. Les services d'éducation publique représentent $80 \%$ de toutes les dépenses liées aux transferts non monétaires et les dépenses pour l'éducation non tertiaire représentent environ deux tiers de tous les transferts non monétaires. En d'autres termes, les dépenses les plus importantes dans le secteur social au Togo sont allouées à l'éducation primaire et secondaire.

L'utilisation du système d'éducation (la scolarisation des enfants) est distribuée de façon progressive : quatre individus sur dix sont scolarisés dans le système d'éducation publique parmi la population la plus pauvre (à l'exclusion des $30 \%$ les plus riches). Le groupe des ménages les plus riches (décile 10) a un taux de scolarisation plus faible dans les institutions publiques : il concerne trois individus sur 10 (Graphique 5). 
Graphique 5. Taux de scolarisation (dans la population générale) au Togo par niveau de scolarité et décile

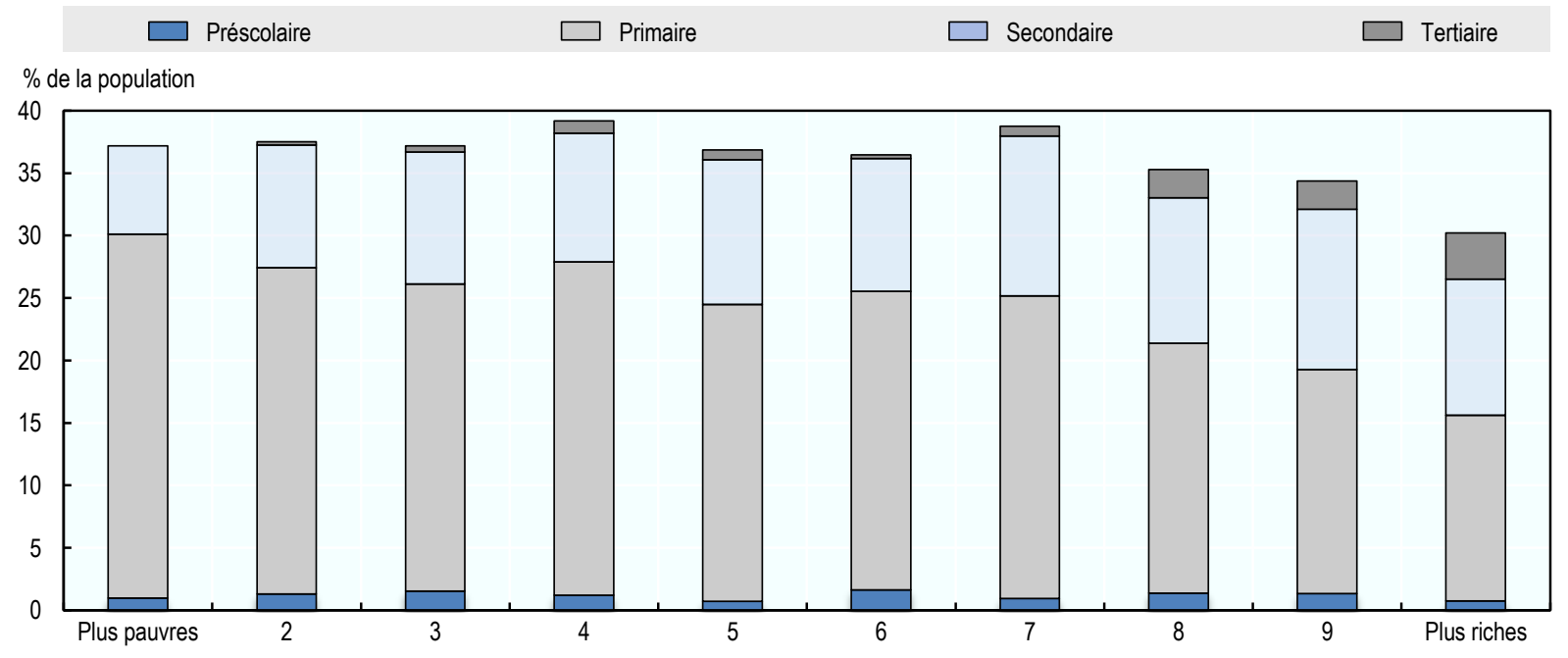

Sources: Calculs des auteurs sur la base du QUIBB 2015 (INSEED, 2015[4]).

La proportion d'individus scolarisés dans le système public aux niveaux secondaire ou tertiaire augmente avec le revenu. Les niveaux d'éducation les plus élevés sont également généralement les plus coûteux. Ainsi, même si le taux de scolarisation dans le public diminue avec le revenu, si les proportions de scolarisation dans des institutions plus coûteuses (secondaire et tertiaire) augmentent, alors les bénéfices de l'éducation publique peuvent ne pas être progressifs dans leur distribution.

En partant de l'hypothèse que les allocations de l'État par écolier dans le pré-primaire représentent $75 \%$ des allocations pour un écolier dans le primaire, et que l'allocation par écolier du secondaire représente $200 \%$ des allocations pour un écolier dans le primaire, alors les dépenses en éducation sont distribuées de façon progressive. Cette progressivité est due aux dépenses dans le primaire, qui affectent la majorité des écoliers scolarisés dans le public. L'éducation tertiaire, qui ne représente qu'une faible proportion des étudiants scolarisés, est régressive (Graphique 6). 
Graphique 6. Courbe de Lorenz (revenu disponible) et courbes de concentration des allocations pour chaque niveau d'éducation au Togo

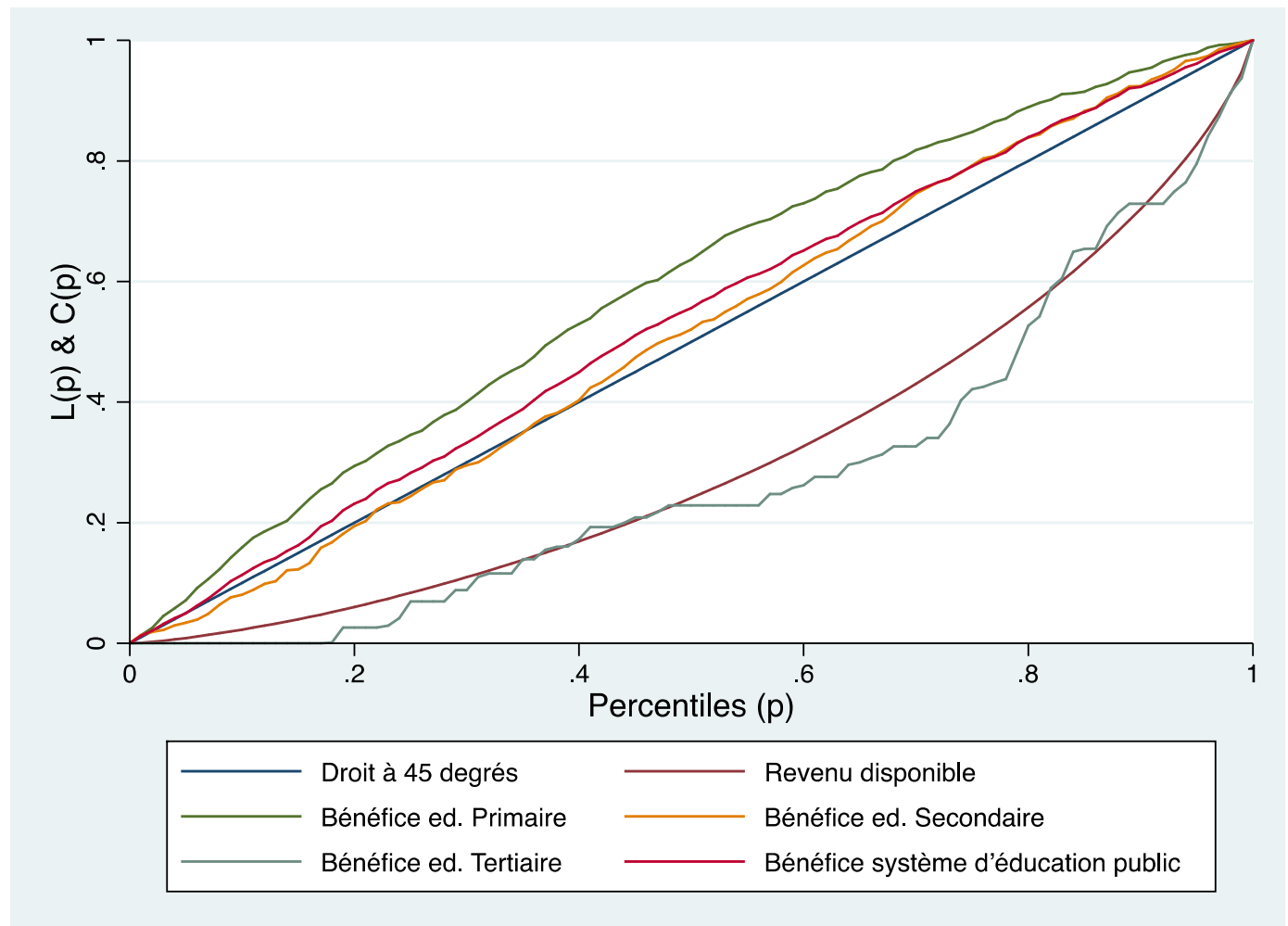

Sources: Calculs des auteurs sur la base du QUIBB 2015 (INSEED, 2015[4]).

Les dépenses liées à l'éducation publique ont un impact similaire sur les inégalités dans d'autres pays africains. Le coefficient d'efficacité d'impact (de la Fuente, Jellema et Lustig, $\left.2018_{[7]}\right)$ de cinq types d'interventions fiscales dans dix pays africains dans les années 2011 à 2015 est présenté dans le Graphique 7. Ce graphique indique la variation de ce coefficient d'efficacité d'impact (barres grises), la moyenne de coefficient (carrés noirs) et le coefficient pour le système fiscal togolais en 2015 (carrés rouges). Ce coefficient résume le niveau maximum théorique de réduction des inégalités de chaque instrument de politique fiscale selon sa taille. Par exemple, la moyenne du coefficient pour les transferts directs est de 0.42. Ceci implique qu'en moyenne, à travers les dix pays et leurs systèmes fiscaux, les dépenses en transferts directs n'atteignent que $42 \%$ de leur impact maximal théorique. Si ce coefficient est négatif, l'instrument de politique fiscale augmente les inégalités au lieu de les réduire. Il peut ainsi être observé que les dépenses en éducation primaire et secondaire au Togo sont plus efficaces que la moyenne, alors que les dépenses en éducation tertiaire réduisent moins les inégalités que la moyenne.

Les impôts directs au Togo sont en moyenne plus efficaces (sur la base du coefficient d'efficacité d'impact) que la moyenne des pays africains de l'échantillon de comparaison. 


\section{Graphique 7. Variation et moyenne du coefficient d'efficacité d'impact par instrument de politique fiscale}

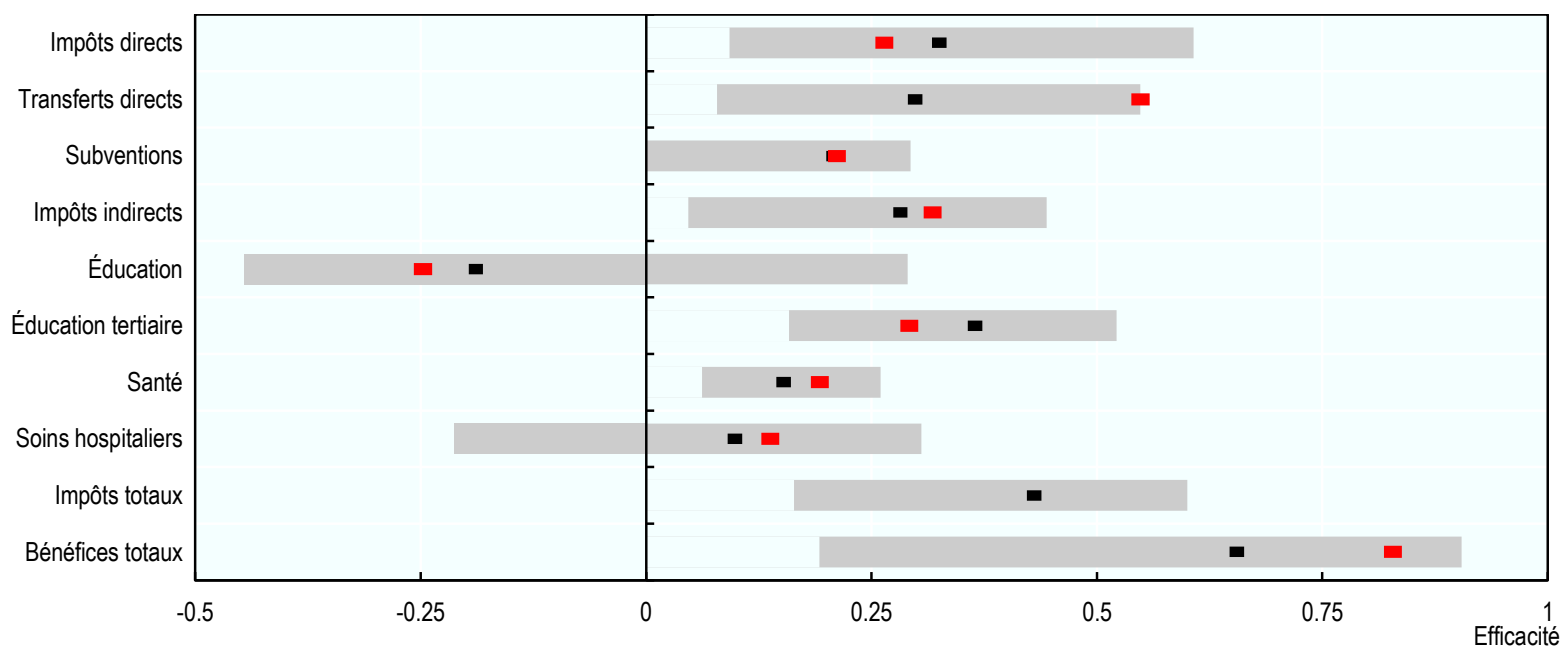

Sources: Enami (2018 $[8])$; de la Fuente, Jellema et Lustig (2018[7]).

Les dépenses en santé représentent environ $13 \%$ des dépenses totales du gouvernement. De même que pour l'éducation, un ménage est considéré comme bénéficiaire de ces dépenses s'il utilise des services de santé. La fréquence de déclaration d'un incident de santé est relativement constante dans les différents déciles de revenus (Graphique 8), ce qui implique l'hypothèse d'une utilisation des services de santé également constante quelle que soit la position des ménages dans la distribution des revenus. Cependant, la somme totale d'utilisation de services de santé, publics et privés, est beaucoup plus importante pour les ménages les plus riches. Les ménages les plus riches choisissent les hôpitaux publics plus souvent que les ménages les plus pauvres $(4.2 \%$ du décile le plus riche utilise des hôpitaux publics en comparaison avec $1.5 \%$ des ménages les plus pauvres), qui choisissent plus souvent les cliniques publiques. 
Graphique 8. Fréquence de déclaration d'un incident de santé et utilisation du système de santé au Togo

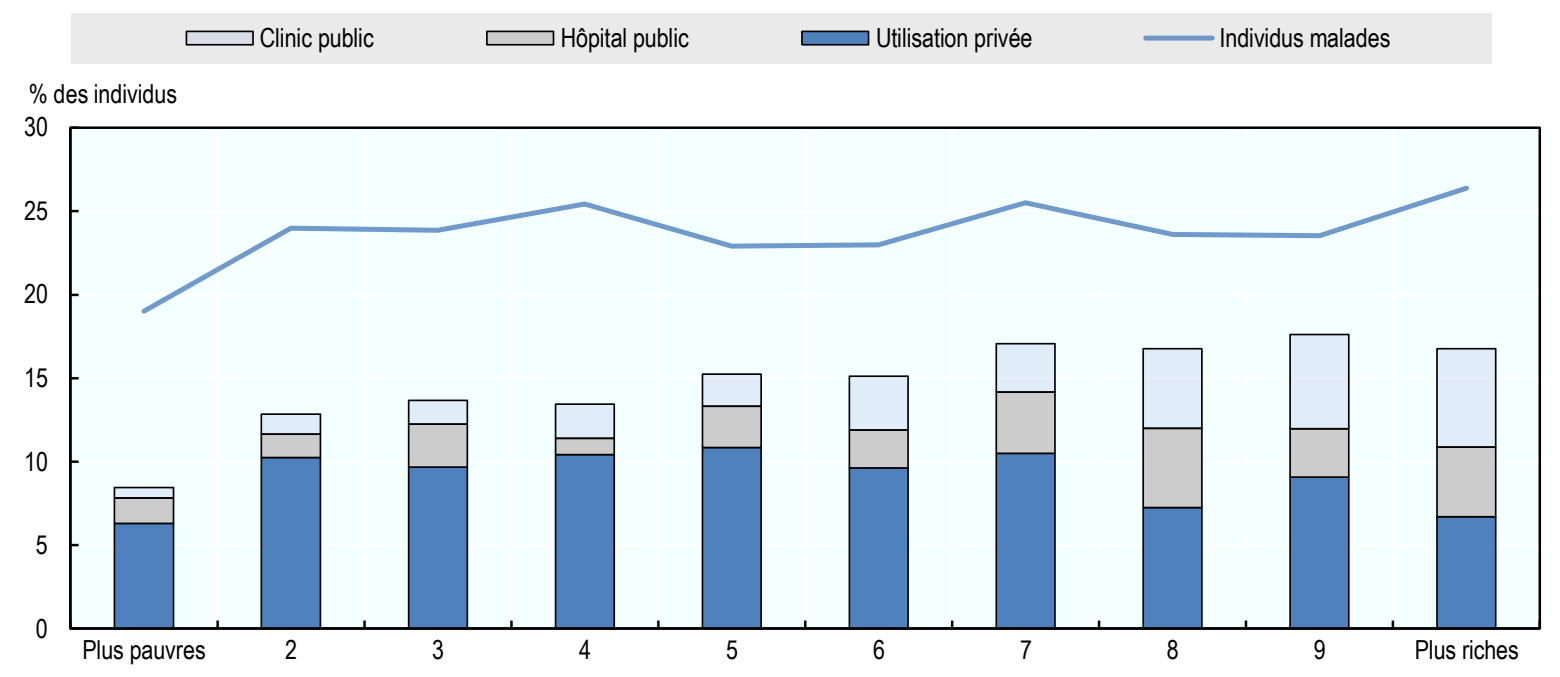

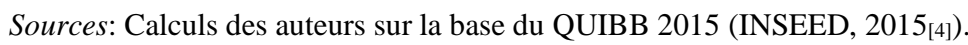

En suivant l'hypothèse qu'une visite dans un hôpital public a un coût deux fois plus important qu'une visite dans une clinique publique, les dépenses totales dans le système de santé public sont distribuées de façon quasiment neutre, proportionnellement. Le Graphique 9 indique que l'impact du système de santé public sur les inégalités au Togo est proche de la moyenne de la région. 
Graphique 9. Courbe de Lorenz (revenu disponible) et courbes de concentration des bénéfices de santé (rang=revenu disponible) au Togo

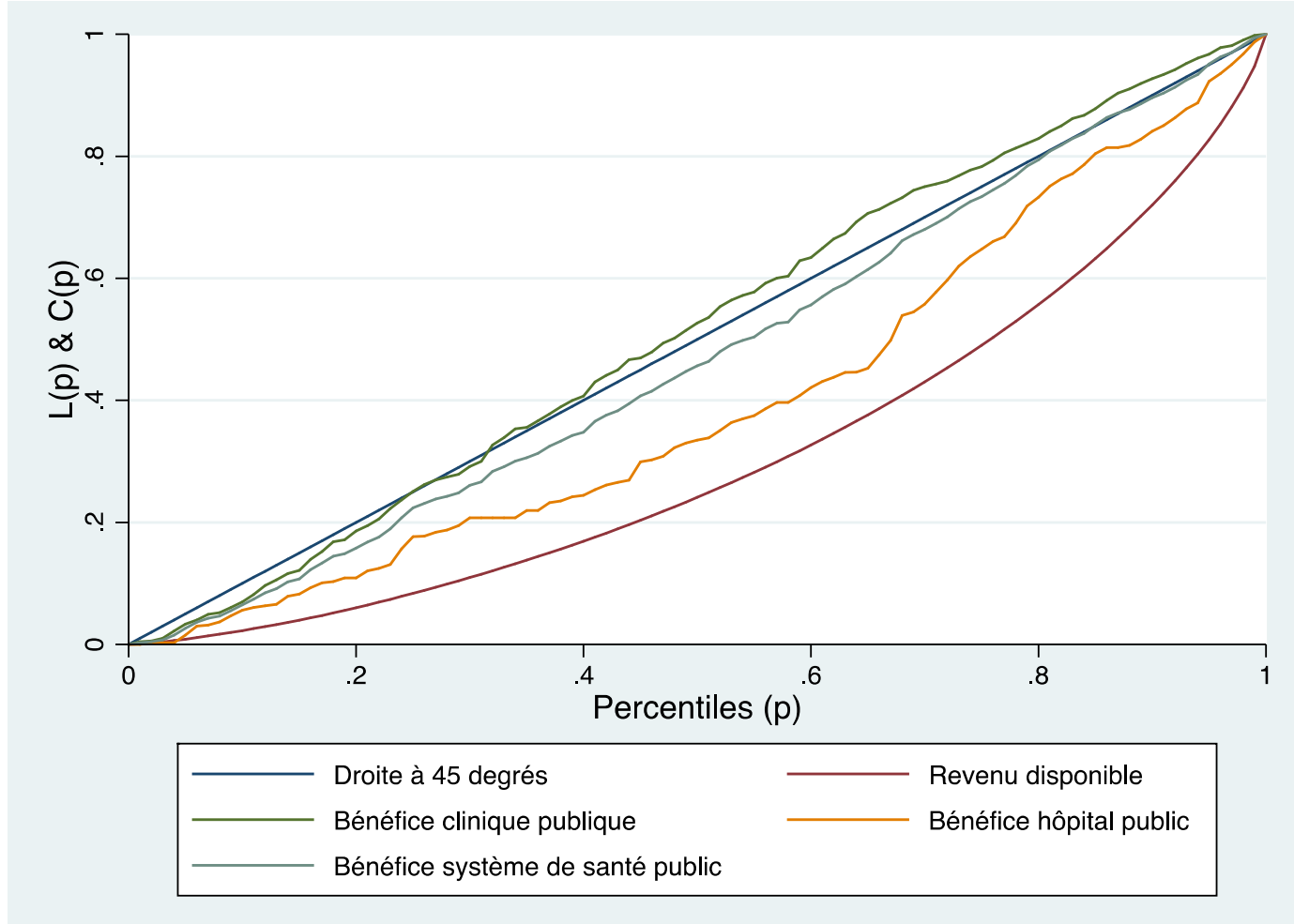

Sources: Calculs des auteurs sur la base du QUIBB 2015 (INSEED, 2015[4]).

En termes de revenus, les bénéfices en éducation et santé publiques sont plus importants pour les ménages les plus pauvres que les bénéfices liés aux subventions en électricité. Ces dernières représentent $0.1 \%$ des revenus (avant subventions) des ménages les plus pauvres (décile 1), alors que les bénéfices en termes d'éducation et de santé représentent environ $4 \%$ de leurs revenus. Les dépenses liées à la TVA sont plus importantes que les bénéfices liés soit à l'éducation, la santé, ou l'électricité pour tous les ménages. Pour les ménages représentant les $70 \%$ les plus riches, la TVA est plus importante que la somme des bénéfices liés à ces trois interventions.

La courbe de Lorenz du Graphique 9 indique la part des ménages dans chaque percentile par rapport à leurs revenus, ainsi que leurs bénéfices en termes de soins de santé. Une distribution parfaitement égalitaire correspondrait à la droite à 45 degrés.

Le Graphique 10 présente l'incidence de tous les transferts évoqués ci-dessus selon les déciles de revenu des ménages. 


\section{Graphique 10. Incidence des transferts, subventions, transferts non monétaires et TVA}

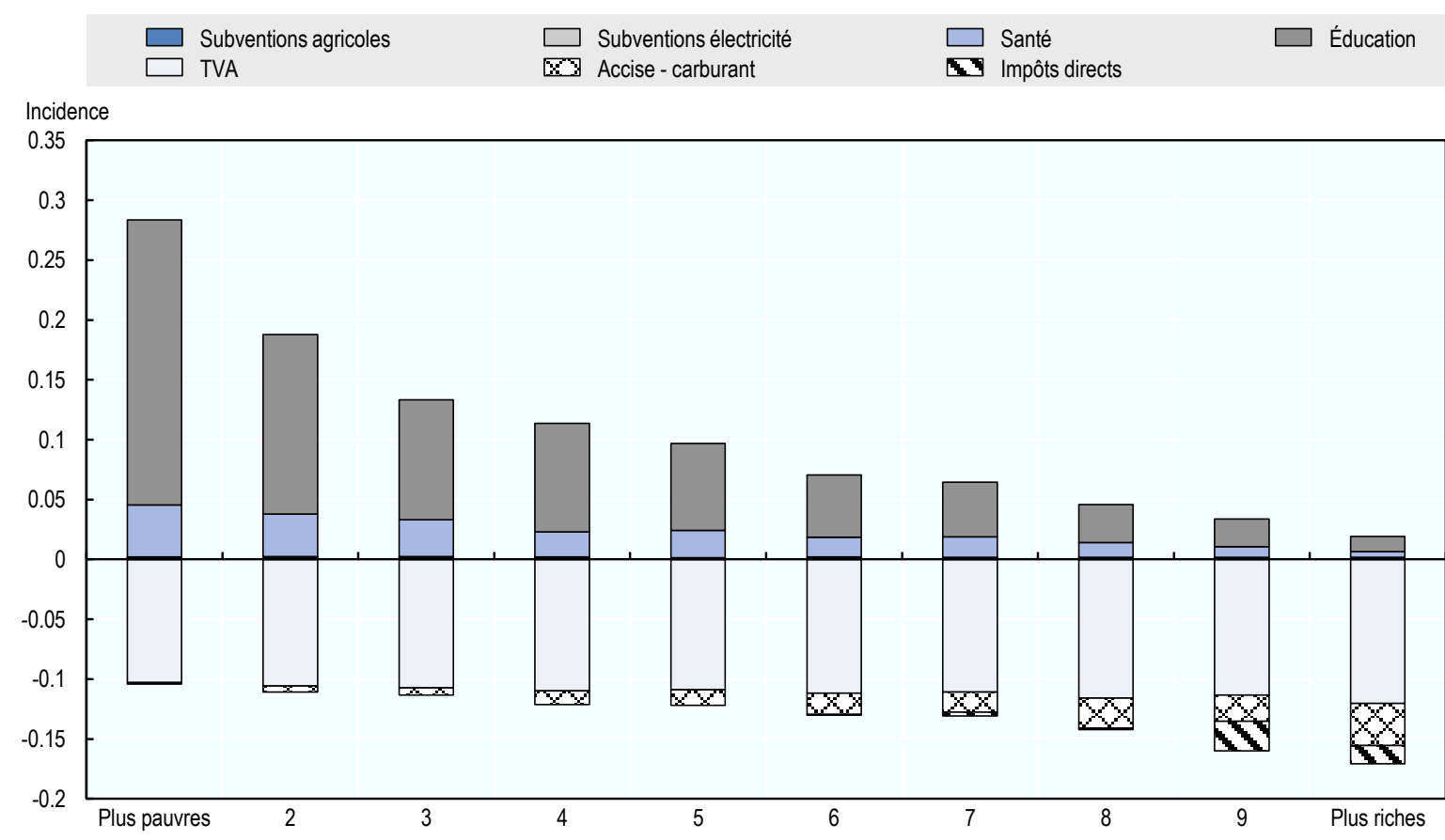

Notes: Représente le pourcentage de revenus pré-transferts et pré-imposition. L'incidence des subventions agricoles est extrêmement faible.

Sources: Calculs des auteurs sur la base du QUIBB 2015 (INSEED, 2015 $[4]$ ).

Le Graphique 11 offre une vue d'ensemble des contributions marginales en points de Gini des différents instruments de politique fiscale sur les inégalités de revenu. 
Graphique 11. Contributions marginales aux inégalités de revenu, par instrument de politique fiscale

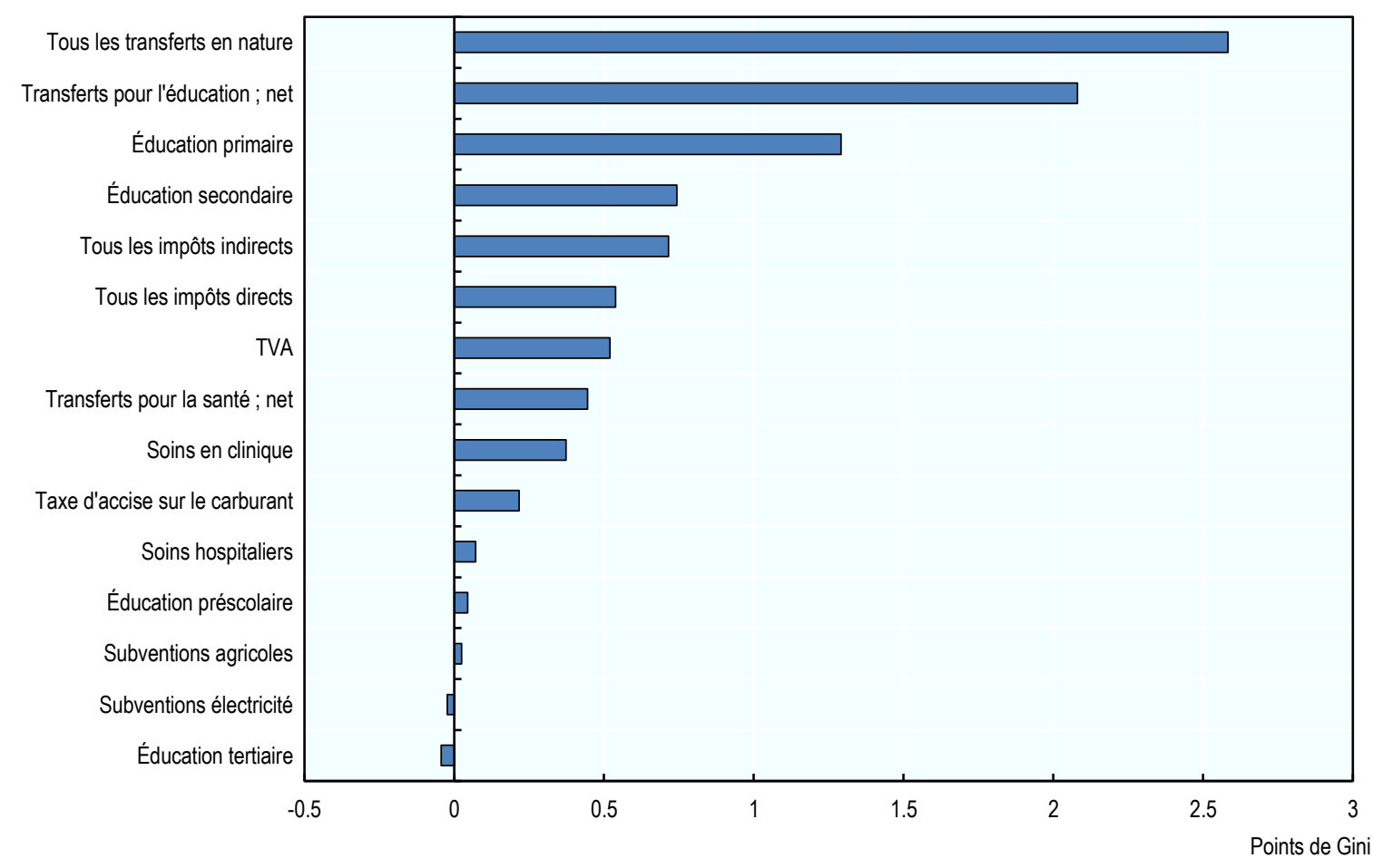

Sources: Calculs des auteurs sur la base du QUIBB 2015 (INSEED, 2015[4]).

\section{Conclusions}

\subsection{La politique fiscale réduit les inégalités}

Les inégalités seraient plus importantes au Togo sans les éléments de politique fiscale pris en compte dans cette étude. Le coefficient de Gini est réduit d'environ 5 points de pourcentage en comparant le revenu pré-fiscal au revenu final. Les instruments de politique fiscale les plus influents dans la réduction des inégalités sont les impôts directs et les transferts non monétaires, en particulier dans les domaines de la santé et de l'éducation. En prenant en compte les subventions en électricité et en engrais pour l'agriculture, et en soustrayant les impôts indirects, on observe un impact sur les inégalités de revenus au niveau national et dans les zones urbaines, mais cet impact est faible dans les zones rurales.

\subsection{La politique fiscale augmente la pauvreté}

Les subventions reçues par les ménages pauvres et vulnérables sont plus faibles que la TVA payée, ils sont donc des payeurs nets dans le système fiscal togolais, contribuant plus aux recettes publiques qu'ils ne bénéficient des dépenses publiques. Le Togo n'a pas à ce jour 
de programme de transferts monétaires de taille suffisante (il couvre moins de $0.5 \%$ de la population) pour protéger les ménages les plus vulnérables. Une politique fiscale incluant une proportion plus large d'impôts directs ainsi que des transferts monétaires de plus grande ampleur pourrait permettre d'éviter un effet appauvrissant sur les ménages les plus vulnérables.

\subsection{Limites de la méthodologie : les transferts non monétaires à travers les services sont importants}

L'éducation et les services de santé sont des avantages sociaux importants, en particulier pour les ménages les plus pauvres. Ces derniers sont en moyenne plus grands et profitent donc proportionnellement plus des avantages sociaux que les ménages les plus riches.

L'accès à l'éducation et aux services de santé publique ne bénéficie cependant pas seulement aux individus en termes monétaires, et le coût de la mise à disposition de ces services n'est pas nécessairement un bon indicateur de la valeur du bénéfice reçu par les utilisateurs des services. Au Togo l'éducation est le poste de dépenses le plus important, et se traduit également par la réduction la plus importante des inégalités tout en bénéficiant aux enfants et étudiants scolarisés à travers une accumulation de capital humain.

Cette étude présente des limitations dues aux choix d'hypothèses sur lesquels elle se base. De plus, bien qu'elle prenne en compte plusieurs aspects de la politique fiscale, tant dans les transferts que dans la taxation des ménages, la méthodologie utilisée présente des limites concernant les services couverts. Il n'est pas possible d'y inclure la dimension de l'accès et de la consommation d'eau au Togo, par exemple. Cette dimension est pourtant importante car le taux d'accès à l'eau est très bas ( $63 \%$ des ménages en moyenne, dont $13 \%$ à travers des branchements privés en 2015). L'État fixe le tarif moyen de l'eau afin d'assurer l'équilibre financier du secteur. Dans un but d'équité et d'accès universel à l'eau, l'État peut mettre en place des subventions pour aider les populations défavorisées. La fiscalité appliquée au secteur de l'eau est actuellement concentrée sur la TVA, avec un taux unique de $18 \%$ s'appliquant aux redevances excédant 10 mètres cubes $\left(\mathrm{m}^{3}\right)$ par mois (les dépenses jusqu'à cette limite sont exonérées). La tarification du secteur de l'eau n'a pas évolué depuis 2001. Une étude récente montre cependant qu'outre la taxation et le maintien de cette première tranche sociale exemptée, des bénéfices en termes d'équité ainsi qu'en termes d'équilibre financier pourraient être obtenus avec la mise en place d'une grille de tarification de l'eau plus graduelle. De plus, la disponibilité des branchements étant particulièrement problématique pour des raisons de coûts, une subvention pour ces deniers permettrait un accès à l'eau plus large aux ménages les plus vulnérables (Artelia, 2016 ${ }_{[9]}$ ). 


\section{Notes}

1. Afrique du Sud, Comores, Éthiopie, Ghana, Sénégal, Mali, Namibie, Ouganda, Tanzanie, Togo, et Zambie.

2. Dans le questionnaire 2015 du QUIBB, tous les ménages reportant des dépenses en électricité sont considérés comme ayant accès à l'électricité. Le pourcentage des ménages reportant l'électricité comme leur principale source de lumière en 2015 est de $85 \%$ dans les zones urbaines et $14 \%$ dans les zones rurales, pour une moyenne combinée de $48.3 \%$.

3. Par exemple, le programme le plus important de transferts monétaires ne comprenait que 14879 bénéficiaires en 2015 , représentant $0.2 \%$ de la population, pour un budget équivalent à 1.3 milliard FCFA (moins de $0.1 \%$ du PIB).

4. L'OCDE (OCDE/ATAF/CUA, 2017 $\left.7_{[5]}\right)$ indique une désagrégation des recettes d'accises pour les produits pétroliers et les autres droits d'accises. Ces derniers représentent environ la moitié des accises, mais ne sont pas détaillés. Ils ne peuvent donc pas être inclus dans cette analyse. 


\section{Références}

Artelia (2016), Élaboration d'une étude tarifaire de l'eau potable en milieu urbain : Rapport final définitif, Programme des Nations Unies pour le développement, New York.

BIT (à paraître), Analyse du système de protection sociale au Togo à travers le processus SPPOT: Vers un socle national de protection sociale au Togo, Bureau International de Travail, Genève.

Causa, O. et M. Hermansen (2017), «Income redistribution through taxes and transfers across OECD countries », OECD Economics Department Working Papers, no. 1453, Éditions OCDE, Paris, http://dx.doi.org/10.1787/bc7569c6-en.

de la Fuente, A., J. Jellema et N. Lustig (2018), Fiscal Policy in Africa: Welfare Impacts and Policy Effectiveness, La Banque Mondiale, Washington, DC.

Lustig, N. (dir. pub.) (2018), Measuring the Effectiveness of Taxes and Transfers in Fighting Inequality and Poverty, Brookings Institution Press and CEQ Institute, Tulane University.

EUSPS et BIT (2018), Social Protection Policy Option Tool Report, Bureau International du Travail, Genève.

FMI (2017), 2016 Article IV Consultation and Request for a three-year arrangement under the Extended Credit Facility - Press releases; Staff Report; and a Statement by the Executive Director for Togo, Fonds Monétaire International, Washington, DC.

INSEED (2015), Questionnaire Unifié des Indicateurs de base du Bien-Être, Institut national de la statistique, des études économiques et démographiques (INSEED), Ministère de la Planification du Developpement, Republique Togolaise, Lome.

Lustig, N. (2016), « Analytic Foundations: Measuring the Redistributive Impact of Taxes and Transfers », dans Commitment to Equity Handbook : A Guide to Estimating the Impact of Fiscal Policy on Inequality and Poverty, Brookings Institution Press et CEQ Institute, Washington, DC, https://ssrn.com/abstract=2893797.

OCDE/ATAF/CUA (2017), Statistiques des recettes publiques en Afrique 2017, Éditions OCDE, Paris, http://dx.doi.org/10.1787/9789264280854-en-fr. 